\title{
Probing the Birth and Dynamics of Hydrated Electrons at the Gold/Liquid Water Interface via a Novel Optoelectronic Approach
}

François Lapointe, Martin Wolf, Kramer Campen, Yujin Tong

Submitted date: $27 / 01 / 2020$ - Posted date: 28/01/2020

Licence: CC BY-NC-ND 4.0

Citation information: Lapointe, François; Wolf, Martin; Campen, Kramer; Tong, Yujin (2020): Probing the Birth and Dynamics of Hydrated Electrons at the Gold/Liquid Water Interface via a Novel Optoelectronic Approach. ChemRxiv. Preprint. https://doi.org/10.26434/chemrxiv.11739819.v1

The hydrated electron has fundamental and practical significance in radiation and radical chemistry, catalysis and radiobiology. While its bulk properties have been extensively studied, its behavior at buried solid/liquid interfaces is still unclear due to the lack of effective tools to characterize this short-lived species in between two condensed matter layers. In this study, we develop a novel optoelectronic technique for the characterization of the birth and structural evolution of solvated electrons at the metal/liquid interface with a femtosecond time resolution. We thus recorded for the first time their transient spectra (in a photon energy range from 0.31 to $1.85 \mathrm{eV}$ ) in situ with a time resolution of $50 \mathrm{fs}$. The transient species show state-dependent optical transition behaviors from being isotropic in the hot state to perpendicular to the surface in the trapped and solvated states. The technique will enable a better understanding of hot electron-driven reactions at electrochemical interfaces.

File list (2)

Solvated Electron_Tong.pdf (0.92 MiB) view on ChemRxiv • download file 


\title{
Probing the Birth and Dynamics of Hydrated Electrons at the Gold/Liquid Water Interface via a Novel Optoelectronic
}

\section{Approach}

\author{
François Lapointe ${ }^{1,2}$, Martin Wolf ${ }^{1}$, R. Kramer Campen ${ }^{1,3}$, Yujin Tong ${ }^{1,3, *}$
}

\section{Affiliations}

1. Fritz Haber Institute of the Max Planck Society, Faradayweg 4-6, 14195 Berlin, Germany

2. Now at: National Research Council Canada, 1200 Montreal Road, Ottawa, Ontario K1A 0R6, Canada

3. Faculty of Physics, University of Duisburg-Essen, Lotharstrasse 1, 47057 Duisburg, Germany

* Correspondence author: tong@fhi-berlin.mpg.de

\begin{abstract}
The hydrated electron has fundamental and practical significance in radiation and radical chemistry, catalysis and radiobiology. While its bulk properties have been extensively studied, its behavior at buried solid/liquid interfaces is still unclear due to the lack of effective tools to characterize this short-lived species in between two condensed matter layers. In this study, we develop a novel optoelectronic technique for the characterization of the birth and structural evolution of solvated electrons at the metal/liquid interface with a femtosecond time resolution. We thus recorded for the first time their transient spectra (in a photon energy range from 0.31 to $1.85 \mathrm{eV}$ ) in situ with a time resolution of $50 \mathrm{fs}$. The transient species show state-dependent optical transition behaviors from being isotropic in the hot state to perpendicular to the surface in the trapped and solvated states. The technique will enable a better understanding of hot electron-driven reactions at electrochemical interfaces.
\end{abstract}




\section{Introduction}

The hydrated electron is the most fundamental ion in solution[1], a metastable structure of water molecules interacting with a free electron. In bulk water, the hydrated electron's lifetime is relatively long and reaches up to microseconds in span [2], but its strong reducing potential renders it highly reactive, and thus short-lived, in the presence of electron acceptors. Consequently, hydrated electrons can damage DNA, create radicals and induce a plethora of reductive reactions. Electrons in solution have been extensively studied from the standpoint of radiobiology, radical and radiation chemistry, and charge transfer systems for their importance in radiotherapy, physiology, catalysis and atmospheric reactions [3-6].

The hydrated electron structure and dynamics from inception to solvation are well known in the bulk [7-11], in clusters [12-14], and at the metal/thin amorphous ice interface [15-17]. Work is also underway to better understand its behavior at the air/water interface [18-21]. In bulk water, the generally accepted model proposes that a 4- to 6-molecule solvation shell hosts the electron in a cavity $[22,23]$ with a radius of gyration $\left(r_{g}\right)$ of $\sim 2.45 \AA$ [1]. A contested alternative noncavity model suggests that the bulk hydrated electron resides in a volume of increased water density [24]. The hydrated electron at the metal/liquid water interface is, however, much less well understood presumably because of the lack of suitable experimental characterization tools for such an environment.

Assuredly, the electrified metal/electrolyte interface $[25,26]$ is highly relevant to technological systems—e.g. batteries, fuel cells, Grätzel's cells, etc.-that rely on heterogeneous electron transfer (HET) $[27,28]$ to store and convert energy or to carry out electrochemical reactions. "Improving such devices requires to understand the mechanism by which the electron moves across the solid/liquid interface. However, such a task is extremely challenging as the charge transfer dynamics are exceedingly short, on the femto- to picosecond time scale. Conventional electrochemical methods, such as those based on potential sweep or impedance measurements, may access the time scale of tens of nanoseconds to the best [29, 30], which may be sufficient to address the mass diffusion problems. They are, however, unfit to provide the desired dynamical information on ultrafast time scales ( $\ll$ picoseconds), on which hot electron relaxation, solvent reorganization and photoexcited isomerization take place. To study these processes, an integration of ultrashort laser pulses and electrochemical control has the 
potential to grant access to the relevant time scales, provided that several significant obstacles can be resolved.

From an experimental perspective, elucidating the mechanism of electron transfer under steady-state nonequilibrium conditions is generally not possible: a perturbation experiment is in fact required, in which the electron transfer is triggered at a well-defined time and the electrochemical interface is characterized with a high time resolution as the system returns to steady-state[31]. However, performing such an experiment with a conventional electrical detection scheme also has a limited time resolution, which is set by the $R C$ value of the system, i.e., $\gg$ nanoseconds, regardless of the electrical or optical trigger length [32-37].

To investigate with higher time resolution the ejection of an electron from a metal and the formation of a solvated electron, several groups have characterized model systems in UHV, i.e., solids with few monolayers of ice, using femtosecond optical pulses to initiate the electron transfer and femtosecond-resolved photoemission measurements as a probe [38, 39]. While this approach on model systems yielded important and interesting developments, we need to perform measurements with a similar time resolution under operando conditions to appropriately characterize the reactions at the electrified electrochemical interface. Unfortunately, the thick liquid layer covering the buried interface precludes direct application of UHV surface science techniques in which electrons or atoms are probes. Moreover, purely optical techniques often suffer from interfering signals from the metal surface charge dynamics: the pump pulse both perturbs the metal surface electronic structure and causes an electron transfer, making the deconvolution from the sought-after signal difficult. In part because of those challenges, there are no reports of the spectral observables that offer insight into the structure of the hydrated electron at electrochemical interfaces.

We have developed a surface-sensitive optoelectronic technique for the synthesis and detection of hydrated electrons at the electrode/electrolyte interface that gives access to the interfacial dynamics from birth to the solvation of the electron species. This method reveals not only the transient spectra of different interfacial hydrated electron states, from the terahertz/mid-infrared (THz/MIR) to the near-infrared (NIR) spectral region, but also the polarization properties of the transient species. Because the method demonstrated here is quite general, we expect it to find wide applicability in the study of the structure and dynamics of short-lived ionic species at electrochemical interfaces. 


\section{Experimental Section}

Details of the ultrafast photovoltage measurement scheme can be found in the Supp. Info. In brief, through excitation by a repeated ultrashort UV pulse $(1 \mathrm{kHz}, 267 \mathrm{~nm})$ with a fluence of $\approx 0.6 \mathrm{~mJ} / \mathrm{cm}^{2}$, electrons are ejected from the electrode into the solution using the photoelectric process. This creates a photovoltage $\Delta V_{1}$, which can be measured using a conventional potentiostat, that reaches a stationary value after $20-30 \mathrm{~min}$ as governed by mass transport and the cell geometry. A second pulse of lower photon energy (same repetition rate) is used to perturb the stabilized system at a variable ultrashort time delay. A photovoltage change $\Delta V_{2}$ is induced that depends strongly on the photon energy of the $2^{\text {nd }}$ pulse and the delay between the two pulses. Measuring and extracting $\Delta V_{2}$ for a series of delay values yields a trace reflecting the ultrafast hydrated electron dynamics at the electrode interface.

The UV pulse at $267 \mathrm{~nm}(4.64 \mathrm{eV})$ is generated by tripling a $800 \mathrm{~nm}(1.55 \mathrm{eV})$ pulse in a $\beta$-barium borate (BBO) crystal. The Gaussian full width at half maximum (FWHM) of the UV pulse is $\approx 110 \mathrm{fs}$ after transmission through the lens, window and water layer overlying the sample (see Supp. Info.) Various combinations of an optical parametric amplifier (OPA) and a noncollinear difference frequency generation accessory (nDFG) were used to obtain pulses of different photon energies for the second pump (see the Supp. Info. for details). The second pump pulses had an estimated Gaussian FWHM of $\sim 80 \mathrm{fs}$. The second laser pulse is delayed with respect to the first pulse by times ranging from -4 to 100 ps, such that a full delay trace is created at every photon energy. The UV-induced bleaching of the nonresonant sum-frequency generation (SFG) signal of the gold surface, generated by mixing a $1.55 \mathrm{eV}$ beam with the second pump beam, is used to define the position of time zero.

The spectroelectrochemical cell is depicted in Fig. S2 of the Supp. Info. Three electrodes (auxiliary, pseudo reference and working electrode) were patterned on a quartz plate of $45 \mathrm{~mm}$ in diameter by electron beam evaporation resulting in a $5 \mathrm{~nm}$ chromium adhesion layer overlain by $200 \mathrm{~nm}$ of gold. Copper foil stripes electrically connected the gold pads

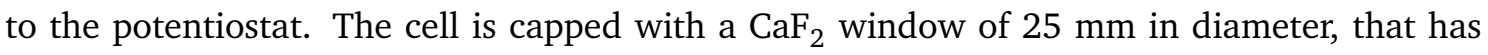
been drilled with two holes to allow for the flow of electrolyte $(\sim 6 \mu \mathrm{L} / \mathrm{s}$, maintained by a peristaltic pump). A spacer of polytetrafluoroethylene (PTFE, $50 \mu \mathrm{m}$ thick) sets the height of the cell's inner chamber for a volume of approximately $6 \mu \mathrm{L}$, such that absorption of light by the liquid phase is minimized. For the electrolyte, we used a solution of $0.5 \mathrm{M} \mathrm{Na}_{2} \mathrm{SO}_{4}$ in 
A

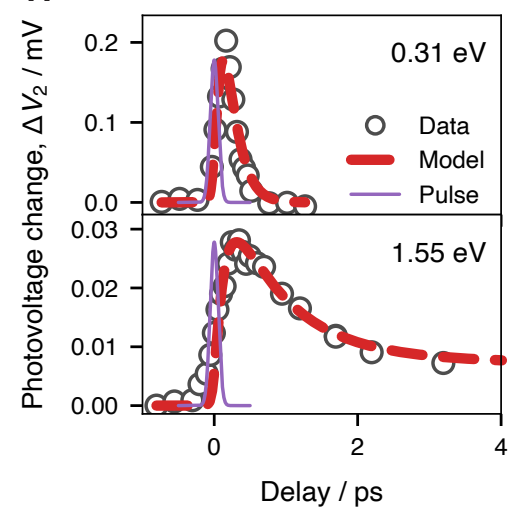

B

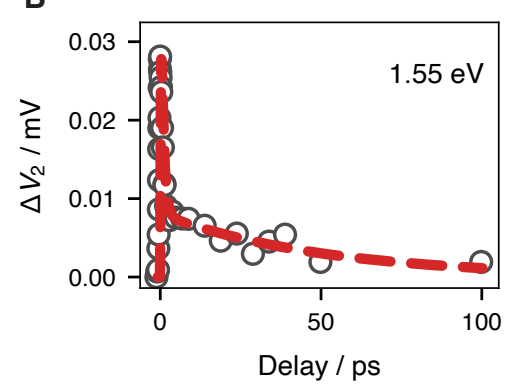

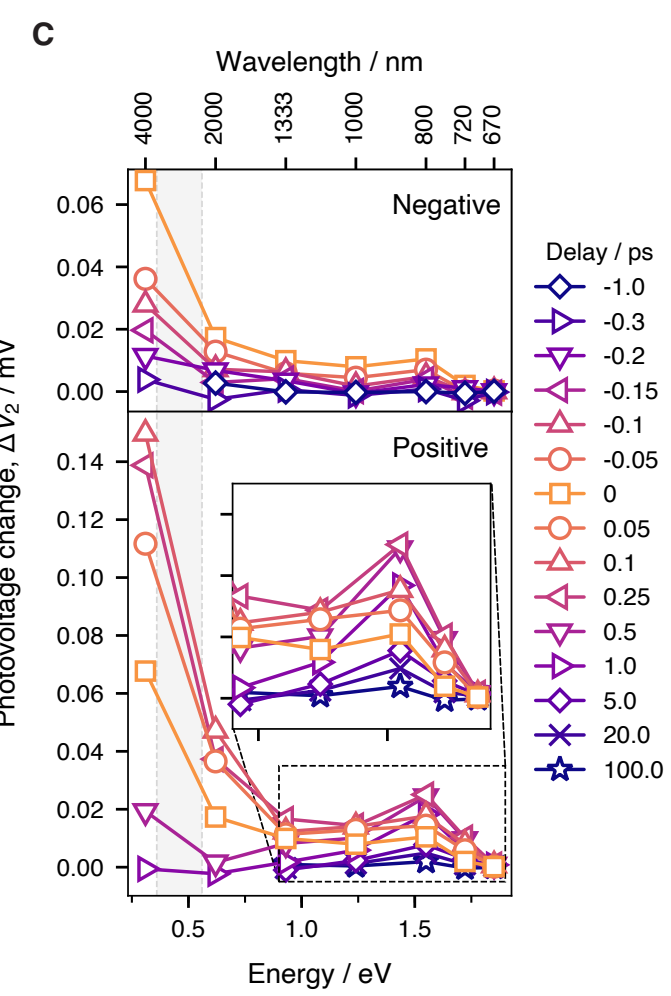

Figure 1: (a-b) The photovoltage change $\Delta V_{2}$ versus the delay time. (a) At delay times smaller than 4 ps. (b) At longer delay times, up to 100 ps. Red dashed traces show the best fit of the three-level hydrated electron model, while the thin blue line show the position and width of the UV pump pulse. (c) The photovoltage change $\Delta V_{2}$ sliced at different delay times, presented against the second pump energy. Top panel: At negative delay times. Bottom panel: Positive delay times. Inset: Zoom of the region around the shoulder at $1.55 \mathrm{eV}$. The gray bands delimit a region where Laenen et al. found strong mid-infrared absorption bands due to $\mathrm{e}^{-}:\left(\mathrm{H}_{2} \mathrm{O}\right)_{\mathrm{n}}$ and $\mathrm{OH}: \mathrm{e}^{-}$precursors [40].

deionized water (18.2 M $\Omega \cdot \mathrm{cm}$, Millipore), degassed by bubbling $\mathrm{N}_{2}$ for at least 30 min prior to the experiment. The photoinduced electrode potential was measured in open circuit mode (open circuit potential, OCP) and corrected for the pulse energy of the first and second pulses and water absorption coefficient at the second pulse wavelength (see Supp. Info.)

\section{Results and Discussion}

Fig. 1(a) presents the photovoltage change $\Delta V_{2}$ (induced by the $2^{\text {nd }}$ laser beam) as a function of the time delay between the two pulses for a range $<4$ ps. At a second pulse photon energy of $0.31 \mathrm{eV}[4 \mu \mathrm{m}$ in wavelength, upper panel of Fig. 1(a)], we observe a rapid rise, but the signal decays almost as fast reaching a null intensity in about $500 \mathrm{fs}$. As we tune to higher second pulse photon energies, e.g., $1.55 \mathrm{eV}$ [800 nm in wavelength, lower panel of Fig. 1(a)], the rise is delayed by 50-100 fs, the signal decays much slower and it persists for tens of picoseconds 
[Fig. 1(b)]. The dynamics traces for all second pulse photon energies used are shown in the Supp. Info. (Fig. S6).

Fig. 1(c) shows the transient, coarse spectral profile of the response of the electrode/electrolyte interface excitation from its inception to its stabilization with the change in photovoltage, $\Delta V_{2}$ plotted as a function of the energy of the second pump. The figure is created by slicing the dynamics data for a set of representative delays, with negative delays in the upper panel and positive delays in the lower panel. Before the arrival of the first pump pulse (e.g., at -1 ps), we observe a null response (diamonds, dark blue). The onset of the UV-induced excitation is marked by the rapid rise of a component in the mid-infrared region that culminates at $\sim+0.1$ ps [upward facing triangles, orange, Fig. 1(c), lower panel]. The rise is followed by a signal decay that is almost as fast, reaching a null intensity in less than 1 ps. This component is a broad feature whose maximum intensity clearly lies at photon energies below our measurement window. Similar spectra in the far- to mid-infrared were observed previously in bulk water experiments $[7,11,41]$ and attributed to photon absorption of delocalized excess electrons prior to localization.

The broad feature also shows a shoulder that concomitantly appears at $\sim 1.55 \mathrm{eV}(800 \mathrm{~nm})$ [inset of Fig. 1(c)], continues to grow until approximately $+0.25 \mathrm{ps}$, and then starts to slowly decay. A small residual of the shoulder is still observable even after $100 \mathrm{ps,} \mathrm{while} \Delta V_{2}$ for the other energies has already returned to zero. The feature appears at energies close to, but slightly lower than, the reported $p \leftarrow s$ transition of the solvated electron at $1.72 \mathrm{eV}(720 \mathrm{~nm})$ in bulk liquid water [7], and much lower than gold's interband transitions (2.38 eV) [42].

In a simplified portrait, the delocalized hot electron population in the water conduction band would yield a Drude-like optical response in the THz/MIR region of the electromagnetic spectrum, and hydrogen-like localized electrons would behave as particles-in-a-box, with the $p \leftarrow s$ transition resonant in the near-infrared region. The low and high energy features in our experimental spectra [Fig. 1(c)] thus closely coincide with the optical transitions of the bulk localized solvated electron and its precursor states $[7,8,11,43,44]$. Thereby, here we propose that the resonant species at the electrode/electrolyte interface is the hot electron synthesized by the UV pulse, and that we follow the localization and relaxation to the solvated state.

In our experimental configuration, we envision two ways in which incident photons can be transduced into a photovoltage: by heating the metal and creating a temperature gradient at the metal/solution boundary (such as in temperature jump measurements [45- 
47]), or by injecting uncompensated charges in the electrical double layer (EDL), which acts as a capacitor [32]. In order to prove that the observed photovoltage $\Delta V_{2}$ is related to photoinjection and not to the metal film heating, we conducted an experiment where the UV (4.64 eV, $267 \mathrm{~nm}$ ) first pump was replaced by a blue $(3.10 \mathrm{eV}, 400 \mathrm{~nm})$ pump beam of equal power such that photoinjection would be attenuated, while a hot nonequilibrium electron population would still be generated in the metal [Fig. 2(a)][42]. In the metal film heating scenario, the effects of the two colors on the system should not differ dramatically. The $267 \mathrm{~nm}$ pumped signal is displayed in the lower panel of Fig. 2(b), where a photovoltage increase of $7-9 \mathrm{mV}$ is observed when the shutter of the second pump beam $(0.31 \mathrm{eV})$ is open. In stark contrast, the $400 \mathrm{~nm}$ pumped signal (upper panel) is barely modulated by the second pump shutter actuation. As sketched in Fig. 2(a), we deduce that metal electrons excited by $3.10 \mathrm{eV}$ photons are unable to efficiently reach the water conduction band, or any other solution-side acceptor states, while UV pumped electrons are imparted with enough energy to do so and thus that the measured photovoltage arising from the spatial and temporal overlap of two pulsed beams is result of photoinjected charges in solution.

We further argue that the observed change in photovoltage $\Delta V_{2}$ (Fig. 1) caused by the second pump beam involves a resonant excitation at the electrochemical interface subsequent to the photoinjection produced by the first pump beam. Other potential contributions (such as further photoinjection from the electrode, photoejection of charge carriers from the aqueous interface to the electrode, and also the metal's optical properties thermomodulation) can be ruled out from the $2^{\text {nd }}$ pump photon energy-dependent dynamics, relative signs of the signal, as well as the power- and polarization-dependent results (see the Supp. Info. for details). Accordingly, the second pulse photons are absorbed by species located at the interface with a cross section that is proportional to the transition probability. The relaxation back to ground state then releases the extra energy to the solvent and thus locally raises the temperature, inducing a photovoltage from the temperature gradient at the metal/solution boundary.

Our results suggest that we measure for the first time the transient spectra of the hydrated electron and its precursors at the gold/water interface using the two-photon excitationphotovoltage detection scheme. Because the structure, structural dynamics and relative permittivity of electrolyte solutions at an electrode boundary differ dramatically from those of the bulk solution, one might expect that the solvated electron's dynamics and structure should differ as well. Indeed, as shown below, our optoelectronic technique reveals one such 
A

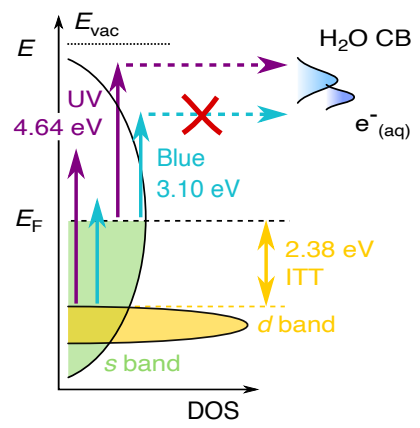

B

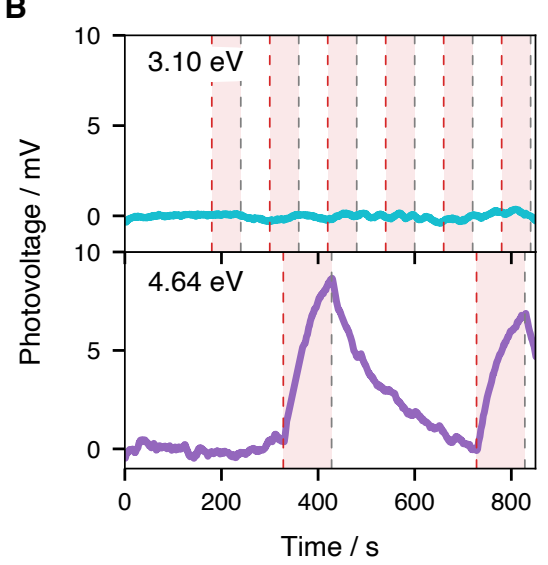

Figure 2: (a) Schematics of gold's DOS and water's CB with the optical transitions enabled by $\mathrm{UV}(4.64 \mathrm{eV})$ and blue $(3.10 \mathrm{eV})$ photons. (b) Comparison of the photovoltage when the first excitation pulse is changed from UV (bottom) to blue (top). The second pulse's photon energy is $0.31 \mathrm{eV}$ in both cases. A polynomial baseline has been removed from the traces to facilitate comparison. The red stripes show the times during which the shutter is open and the second pulse impinges on the electrode. 


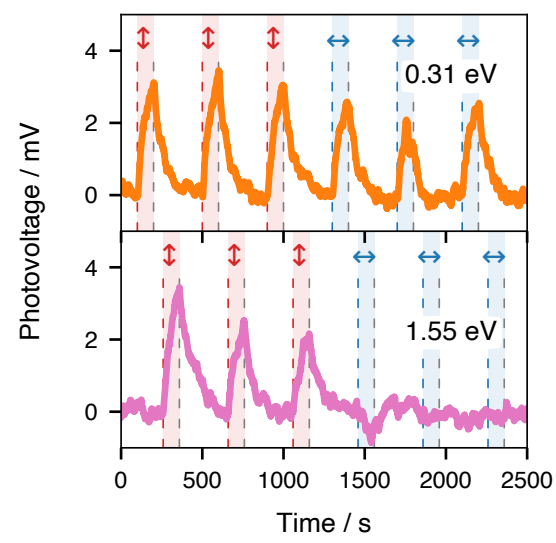

Figure 3: Comparison of the photovoltage with respect to incident polarization of the second pulse for two energies ( 0.31 and $1.55 \mathrm{eV}$ ) at a time delay close to 0 with a first excitation pulse energy of $4.64 \mathrm{eV}$ and $p$ polarization. A baseline has been removed from the traces to facilitate comparison. Vertical double arrows indicate that the second pulse incident polarization is $p$, and horizontal, $s$. The red and blue stripes show the times during which the shutter is open and the second pulse impinges on the electrode.

difference with the bulk properties: different states of the hydrated electron actually display distinct polarization properties when interacting with light.

To elucidate the interaction of the transient species with the substrate, we conducted polarization dependence measurements of the photovoltage induced by the second pump beam at 0.31 and $1.55 \mathrm{eV}$ photon energies. The delay condition was set independently in order to maximize the signal for each second pump beam photon energy $(\sim 0$ at $0.31 \mathrm{eV}, \sim+0.25 \mathrm{ps}$ at $1.55 \mathrm{eV})$. As shown in Fig. 3, a small change $(\approx 1 \mathrm{mV})$ of $\Delta V_{2}$ is observed for the $0.31 \mathrm{eV}$ laser upon the polarization change from $p$ (red vertical arrows) to $s$ (blue horizontal arrows). In comparison, $\Delta V_{2}$ measured in the same conditions, but for a second pump beam at $1.55 \mathrm{eV}$ (lower panel), is almost entirely attenuated in the $s$ polarization state, with a possible small negative component appearing upon the change of polarization. The disparity between these experiments can be rationalized as the nature of the optical transitions taking place at the metal surface for different types of interfacial species as will be discussed in detail below. However the presence of an anisotropic response contrasts strongly with the isotropic feature observed for equilibrated hydrated electrons in the bulk (a response typically attributed to the sphericity of the cavity [48]).

The hydrated electron properties at electrochemical interfaces have been discussed early on [49] after the report of the solvated electron's bulk spectrum [43], but limited by the available tools, conclusions were largely speculative. Here, combining femtosecond laser pulses and photovoltage measurement, we measured the transient spectra of interfacial 


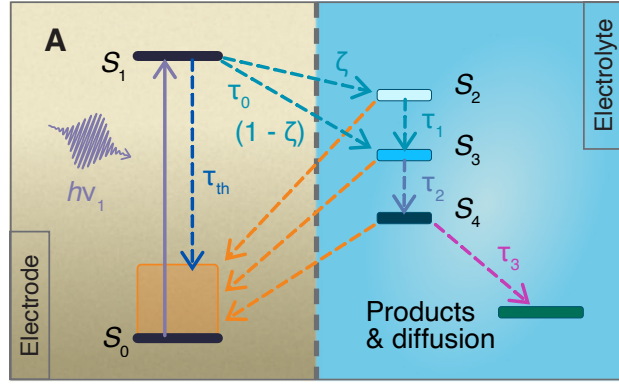

B

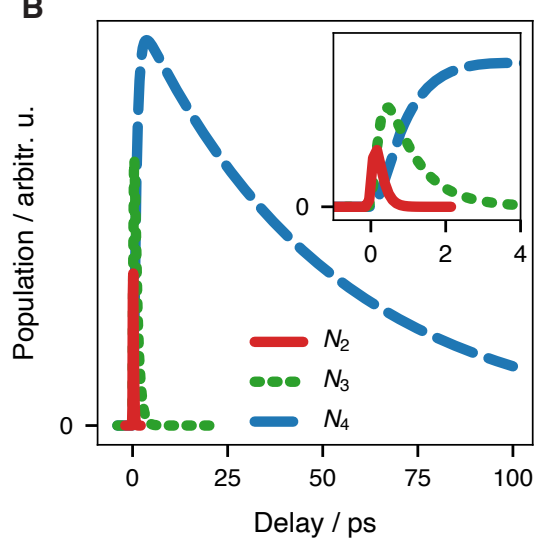

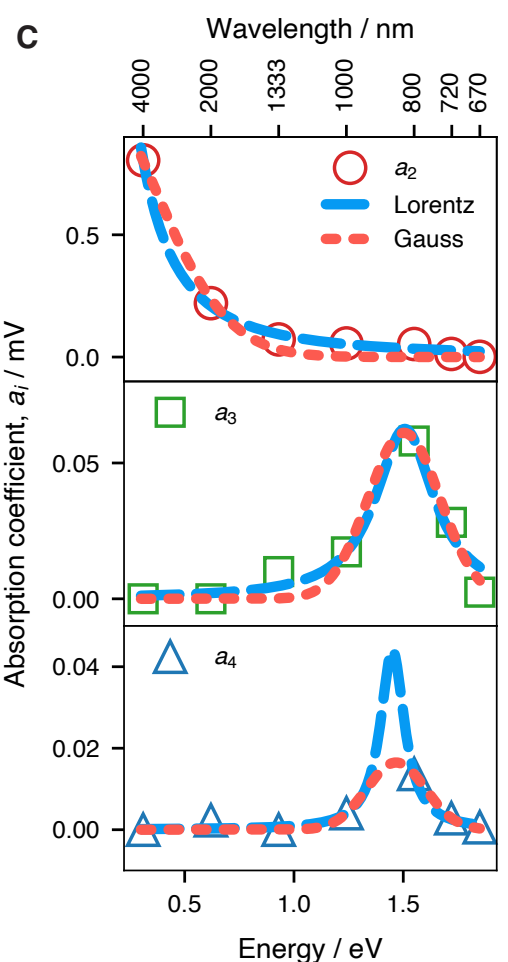

Figure 4: (a) Schematics of the proposed molecular kinetics model. (b) Evolution of hot, trapped and solvated electron populations. Inset: Zoom near origin. (c) The absorption coefficient for the three electron populations in solution.

hydrated electrons and their polarization dependence, hence providing essential information to establish a molecular picture of the hydrated electron's evolution from synthesis to decay. The similarities in the spectra of hydrated electron at the electrochemical interface and those in the bulk and at the metal/ice interface in UHV support a similar relaxation process to the ones in those systems, with intriguing differences described below. In brief, at the metal/ice interface the first step in the photoinjection of electrons from the metal is the creation of hot electrons, right after the transfer, while a small proportion may directly reach preexisting traps [15]. From that hot electron population, a large portion will rapidly return to the electrode $(\sim 85 \%[50])$, but the remaining fraction $(\sim 15 \%)$ is now in a delocalized state in the aqueous medium. In bulk liquid water, the hot electron will first localizes in an excited $p$ state and subsequently relaxes via an internal conversion (IC) step to a modified $s$ state in a nonadiabatic manner [14]. Reorganization of the surrounding solvent molecules completes the relaxation.

Following these works, here we also adopt a three-level hydrated electron model to fit our results that is depicted in Fig. 4(a) and is fully described in the Supp. Info. Tentatively, the state $S_{0}$ is assigned to gold's ground state "Au", $S_{1}$ to gold's nonequilibrium athermal state "Au*", $S_{2}$ 
to hot electrons in the water conduction band "CB", $S_{3}$ to hydrated electrons in the trapped state " $\mathrm{e}_{(\text {trap })}^{-}$" and $S_{4}$ to the solvated state " $\mathrm{e}_{(\mathrm{aq})}^{-}$". Electrons are injected from the $\mathrm{Au}^{*}$ state into the electrolyte with a time constant $\tau_{0}$. The great majority of these electrons reach the CB state. A minor fraction $(1-\zeta)$ is directly injected to the $\mathrm{e}_{(\text {trap })}^{-}$state (wet electron) $[15,17]$; we estimate $\zeta$ to be approximately 0.98. Although there is currently no report of preexisting trap states in liquid water, the appearance from time zero of the shoulder at $1.55 \mathrm{eV}$ in Fig. 1(c) suggests that a similar process happens in the liquid phase at the interface. This is plausible since it is known that the hydrogen bond lifetime in bulk liquid water is $\sim 2.5$ ps [51]. While interfacial water molecules may perform libration motions during the $250 \mathrm{fs}$ initial period in which the peak intensity at $1.55 \mathrm{eV}$ continuously grows, it seems unlikely that significant changes would occur in the water network structure. Finally, the hot, trapped and solvated electron populations are related to the measured signal $\Delta V_{2}$ through absorption coefficients, $a_{i}$ with $i=2,3,4$.

The results of the fits are shown in Fig. 1(a) and (b) as the red dashed lines and in the Supp. Info (Fig. S6). The model describes well the experimental data at all energies for a common set of parameters. The sharp initial transient is mainly caused by the hot and trapped electron populations in the electrolyte [red and green traces, respectively, Fig. 4(b)], while the persisting, slowly decreasing signal is due to the decaying solvated electron population [blue trace, Fig. 4(b)]. The corresponding lifetimes are determined to be $\tau_{1} \sim 140 \mathrm{fs}, \tau_{2} \sim 820 \mathrm{fs}$ and $\tau_{3} \sim 51 \mathrm{ps}$ for IC, solvent reorganization and slow population decay, respectively. As depicted in Fig. 4(a), the solvated electron population $\left(S_{4}\right)$ has two possible loss channels lumped into $\tau_{3}$ : recapture by the electrode or oxidized species from the solution, and transport outside of the EDL into the bulk. In the bulk, the solvated electron diffuses by $\sim 0.3 \mathrm{~nm}$ in 10 ps (diffusion constant $\sim 4.75 \times 10^{-5} \mathrm{~cm}^{2} \mathrm{~s}^{-1}$ ) [52]. This distance being comparable to the solvated electron radius and the average hydrogen bond length, the transport channel's contribution is likely negligible.

Interestingly, the $\mathrm{e}_{(\text {trap })}^{-}$, i.e. $S_{3}$, state lifetime $(820 \mathrm{fs})$ matches the reorientation dynamics of the free (non $\mathrm{H}$-bonded) water $-\mathrm{OH}$ at the air-water interface [53]. Further studies along this line may provide insight into the interface-specific mechanisms of electron localization. From the fits, we also obtained the effective spectra of the hydrated electron at different states [Fig. 4(c)] (see the Supp. Info. for a discussion of the center energy and FWHM of the spectra). The spectrum $a_{2}$ (top panel), which belongs to the state $S_{2}$, can be understood as a Drude- 
like optical transition, while the spectra $a_{3}$ (middle) and $a_{4}$ (bottom), assigned to the states $S_{3}$ and $S_{4}$, respectively, are consistent with $p \leftarrow s$ optical transitions. These physical models explain well the polarization behavior in Fig. 3, where the photovoltage is mostly independent of polarization at a $0.31 \mathrm{eV}$ photon energy, but displays a significant dependence at $1.55 \mathrm{eV}$. This is because Drude-like transitions involving a population uncoupled to the substrate are mostly isotropic, and the $p \leftarrow s$ transitions of populations in proximity with the electrodes display anisotropy due to image dipoles in the metal (dipole cancellation rule).

It is noteworthy that the center energy of the spectral response of the $\mathrm{e}_{(\text {trap })}^{-}$and $\mathrm{e}_{(\mathrm{aq})}^{-}$ species, i.e. the $S_{3}$ and $S_{4}$ states, $(\sim 1.5 \mathrm{eV})$ appears at a significantly lower photon energy than reported in the bulk $(1.7 \mathrm{eV})$. Such a decrease may be due to the displacement of the absolute energy of either or both the ground and excited states of the solvated electron by the proximity of the highly polarizable gold electrode and a low density water network in the presence of the electron. Furthermore, as pointed out above, the dipole cancellation rule at the metal surface allows only the $p_{z}$ suborbital as optically active. The observed lower energy maximum may thus be due to the splitting of the $p$ suborbitals $[48,54,55]$ by solvent polarization and an incomplete solvation shell.

Upon close inspection of the spectra in Fig. 1(c), an isosbestic point at $\sim 1.24 \mathrm{eV}(1 \mu \mathrm{m})$ can be found between 0 and +0.25 ps. This points to the transformation from state $S_{2}$ to states $S_{3}$ and/or $S_{4}$. Does the hydrated electron evolve smoothly from the hot to the solvated states, adopting a continuum of in-between states, or does it jump from one state to another at the gold/liquid water interface? In bulk solution, work by Migus et al. [7], followed by measurements by Eisenthal el al. [56, 57], seemed to support the latter scenario, because an isosbestic point was observed in the transient absorption of the hydrated electron species. Later, a time-dependent shift of the spectral weight from the low to high energy was confirmed first in the NIR $[8,9]$, then in the terahertz [11], while different intermediate species were observed in the MIR [40]. No studies ever showed the evolution of the spectrum in one set of experiments. Our measurements, which give access to a large breadth of the spectrum, suggest that the discrete states model seems also valid at the metal/liquid water interface. Further comparison with bulk data will require better spectral resolution and testing against purely optical sampling.

Further work will address open questions regarding, notably, the actual molecular level changes in the EDL during relaxation, the influence of the electrolyte flow and the tracking 
of the electron as it induces chemistry using a mixture of nonlinear optical and optoelectronic approaches.

\section{Conclusion}

A novel, double optical pump, photovoltage detection method enables the observation of a wavelength-dependent time-resolved response after excitation by an ultrafast UV pulse of a polycristalline gold electrode immersed in an aqueous electrolyte. The spectra provide a window on the interfacial solvated electron and its precursor states. A kinetic model with three electrolyte-side levels, i.e., hot, trapped and solvated electron states, produces a satisfactory fit of the dynamics. Fitting the model to the data yields lifetimes (convoluted with the oxidation by the electrode) of the hot electron of $\tau_{1} \sim 140 \mathrm{fs}$, the trapped electron of $\tau_{2} \sim 820 \mathrm{fs}$ and the solvated electron (at the electrode) of $\tau_{3} \sim 51 \mathrm{ps}$. An isosbestic point near $1.24 \mathrm{eV}$ is observable at short time delays, consistent with the transformation of electrons from a localized state to a trap state. The hot and trapped states exhibit significantly different dipole properties: the hot electron state is isotropic and the trapped electron state is anisotropic with a transition dipole perpendicular to the surface. In contrast with conventional electrical methods, the optoelectronic technique thus provides the means for the study of hot electrondriven processes at the electrified metal/electrolyte interface, and gives access to unique energy- and time-resolved information about charge transfer mechanisms and interfacial chemistry. Granting access to the hydrated electron's birth and stabilization, our approach offers a promising pathway-observing its structure and dynamics evolution with respect to the substrate material and geometry, and the electrolyte nature-to advance the fundamental physics and chemistry of photoelectrocatalysis.

\section{Acknowledgments}

We gratefully acknowledge the technical support from S. Wasle, W. Krauß and S. Kubala. FL thanks the Fonds de recherche du Québec - Nature et technologies (FRQNT) for a postdoctoral scholarship. This study was supported by the European Research Council (ERC) under the European Union's Horizon 2020 research and innovation program (grant agreement no 772286 , to RKC). 


\section{References}

[1] J. M. Herbert, M. P. Coons, Annu. Rev. Phys. Chem. 2017, 68, 447-472.

[2] B. Abel, U. Buck, A. L. Sobolewski, W. Domcke, Phys. Chem. Chem. Phys. 2012, 14, 22.

[3] B. Boudaïffa, P. Cloutier, D. Hunting, M. A. Huels, L. Sanche, Science 2000, 287, 16581660.

[4] B. C. Garrett, D. A. Dixon, D. M. Camaioni, D. M. Chipman, M. A. Johnson, C. D. Jonah, G. A. Kimmel, J. H. Miller, T. N. Rescigno, P. J. Rossky, S. S. Xantheas, S. D. Colson, A. H. Laufer, D. Ray, P. F. Barbara, D. M. Bartels, K. H. Becker, K. H. Bowen, S. E. Bradforth, I. Carmichael, J. V. Coe, L. R. Corrales, J. P. Cowin, M. Dupuis, K. B. Eisenthal, J. A. Franz, M. S. Gutowski, K. D. Jordan, B. D. Kay, J. A. LaVerne, S. V. Lymar, T. E. Madey, C. W. McCurdy, D. Meisel, S. Mukamel, A. R. Nilsson, T. M. Orlando, N. G. Petrik, S. M. Pimblott, J. R. Rustad, G. K. Schenter, S. J. Singer, A. Tokmakoff, L.-S. Wang, T. S. Zwier, Chem. Rev. 2005, 105, 355-390.

[5] K. R. Siefermann, B. Abel, Angew. Chemie - Int. Ed. 2011, 50, 5264-5272.

[6] E. Alizadeh, L. Sanche, Chem. Rev. 2012, 112, 5578-5602.

[7] A. Migus, Y. Gauduel, J. L. Martin, A. Antonetti, Phys. Rev. Lett. 1987, 58, 1559-1562.

[8] C. Pépin, T. Goulet, D. Houde, J.-P. Jay-Gerin, J. Phys. Chem. A 1997, 101, 4351-4360.

[9] V. H. Vilchiz, J. A. Kloepfer, A. C. Germaine, V. A. Lenchenkov, S. E. Bradforth, J. Phys. Chem. A 2001, 105, 1711-1723.

[10] P. Kambhampati, D. H. Son, T. W. Kee, P. F. Barbara, J. Phys. Chem. A 2002, 106, 2374 2378.

[11] J. Savolainen, F. Uhlig, S. Ahmed, P. Hamm, P. Jungwirth, Nat. Chem. 2014, 6, 697-701.

[12] P. Ayotte, M. A. Johnson, J. Chem. Phys. 1997, 106, 811-814.

[13] A. E. Bragg, J. R. R. Verlet, A. Kammrath, O. Cheshnovsky, D. M. Neumark, Science 2004, 306, 669-671.

[14] M. H. Elkins, H. L. Williams, A. T. Shreve, D. M. Neumark, Science 2013, 342, 1496-1499.

[15] C. Gahl, U. Bovensiepen, C. Frischkorn, M. Wolf, Phys. Rev. Lett. 2002, 89, 107402.

[16] J. Stähler, C. Gahl, U. Bovensiepen, M. Wolf, J. Phys. Chem. B 2006, 110, 9637-9644.

[17] J. Stähler, J.-C. Deinert, D. Wegkamp, S. Hagen, M. Wolf, J. Am. Chem. Soc. 2015, 137, 3520-3524.

[18] D. M. Sagar, C. D. Bain, J. R. R. Verlet, J. Am. Chem. Soc. 2010, 132, 6917-6919.

[19] K. R. Siefermann, Y. Liu, E. Lugovoy, O. Link, M. Faubel, U. Buck, B. Winter, B. Abel, Nat. Chem. 2010, 2, 274-279.

[20] J. R. Casey, A. Kahros, B. J. Schwartz, J. Phys. Chem. B 2013, 117, 14173-14182.

[21] K. Matsuzaki, R. Kusaka, S. Nihonyanagi, S. Yamaguchi, T. Nagata, T. Tahara, J. Am. Chem. Soc. 2016, 138, 7551-7557.

[22] L. Turi, P. Rossky, Chem. Rev. 2012, 112, 5641. 
[23] L. Kevan, Acc. Chem. Res. 1981, 14, 138-145.

[24] R. E. Larsen, W. J. Glover, B. J. Schwartz, Science 2010, 329, 65-69.

[25] J. O. Bockris, B. E. Conway, R. E. White, Modern aspects of electrochemistry, Vol. 22, Springer Science \& Business Media, 2012.

[26] O. M. Magnussen, A. Groß, J. Am. Chem. Soc. 2019, 141, 4777-4790.

[27] R. A. Marcus, J. Chem. Phys. 1965, 43, 679.

[28] D. M. Adams, L. Brus, C. E. Chidsey, S. Creager, C. Creutz, C. R. Kagan, P. V. Kamat, M. Lieberman, S. Lindsay, R. A. Marcus, et al., J. Phys. Chem. B 2003, 107, 6668-6697.

[29] A. J. Bard, L. R. Faulkner, J. Leddy, C. G. Zoski, Electrochemical methods: fundamentals and applications, Vol. 2, wiley New York, 1980.

[30] C. R. Bradbury, J. Zhao, D. J. Fermin, J. Phys. Chem. C 2008, 112, 10153-10160.

[31] G. Zwaschka, Y. Tong, M. Wolf, R. K. Campen, ChemElectroChem 2019, 6, 2675-2682.

[32] Y. Y. Gurevich, Y. V. Pleskov, Z. A. Rotenberg, Photoelectrochemistry, Springer US, Boston, MA, 1980.

[33] A. Felske, W. J. Plieth, J. Opt. Soc. Am. B 1986, 3, 815-820.

[34] R. P. Baldwin, S. P. Perone, J. Electrochem. Soc. 1976, 123, 1647.

[35] J. H. Richardson, S. B. Deutscher, A. S. Maddux, J. E. Harrar, D. C. Johnson, W. L. Schmelzinger, S. P. Perone, J. Electroanal. Chem. Interfacial Electrochem. 1980, 109, 95114.

[36] A. Harata, Q. Shen, T. Sawada, Annu. Rev. Phys. Chem. 1999, 50, 193-219.

[37] A. G. Krivenko, J. Krüger, W. Kautek, V. A. Benderskii, Berich. Bunsen. Gesell. 1995, 99, 1489-1494.

[38] N.-H. Ge, C. Wong, R. Lingle, J. McNeill, K. Gaffney, C. B. Harris, Science 1998, 279, 202-205.

[39] K. Onda, B. Li, J. Zhao, K. D. Jordan, J. Yang, H. Petek, Science 2005, 308, 1154-1158.

[40] R. Laenen, T. Roth, A. Laubereau, Phys. Rev. Lett. 2000, 85, 50-53.

[41] N. A. Anderson, K. Hang, J. B. Asbury, T. Lian, Chem. Phys. Lett. 2000, 329, 386-392.

[42] R. W. Schoenlein, W. Z. Lin, J. G. Fujimoto, G. L. Eesley, Phys. Rev. Lett. 1987, 58, 16801683.

[43] E. J. Hart, J. W. Boag, J. Am. Chem. Soc. 1962, 84, 4090-4095.

[44] F. J. Webster, J. Schnitker, M. S. Friedrichs, R. A. Friesner, P. J. Rossky, Phys. Rev. Lett. 1991, 66, 3172-3175.

[45] V. A. Benderskii, G. I. Velichko, J. Electroanal. Chem. 1982, 140, 1-22.

[46] J. F. Smalley, L. Geng, S. W. Feldberg, L. C. Rogers, J. Leddy, J. Electroanal. Chem. 1993, $356,181-200$.

[47] V. Climent, B. A. Coles, R. G. Compton, J. Phys. Chem. B 2001, 105, 10669-10673. 
[48] M. Assel, R. Laenen, A. Laubereau, P. Resolution, J. Phys. Chem. A 1998, 102, 2256-2262.

[49] H. Berg, Electrochim. Acta 1968, 13, 1249-1252.

[50] V. V. Konovalov, A. M. Raitsimring, Y. D. Tsvetkov, Int. J. Radiat. Appl. Instrumentation. Part C. Radiat. Phys. Chem. 1988, 32, 623-632.

[51] H. J. Bakker, J. L. Skinner, Chem. Rev. 2010, 110, 1496-1517.

[52] K. H. Schmidt, W. L. Buck, Science 1966, 151, 70-71.

[53] C.-S. Hsieh, R. K. Campen, A. C. Vila Verde, P. Bolhuis, H.-K. Nienhuys, M. Bonn, Phys. Rev. Lett. 2011, 107, 116102.

[54] D. Borgis, A. Staib, Chem. Phys. Lett. 1994, 230, 405-413.

[55] L. Turi, D. Borgis, J. Chem. Phys. 2002, 117, 6186-6195.

[56] F. H. Long, H. Lu, K. B. Eisenthal, Phys. Rev. Lett. 1990, 64, 1469-1472.

[57] X. Shi, F. H. Long, H. Lu, K. B. Eisenthal, J. Phys. Chem. 1996, 100, 11903-11906. 


\section{Table of Contents}

\section{TOC Descriptive Text}

Two ultrashort laser pulses of different photon energy are used to generate and excite hydrated electrons at the gold electrode / aqueous electrolyte interface. The hydrated electron's ultrafast solvation dynamics can thus be reconstructed with a simple photovoltage measurement.

\section{TOC Graphic}

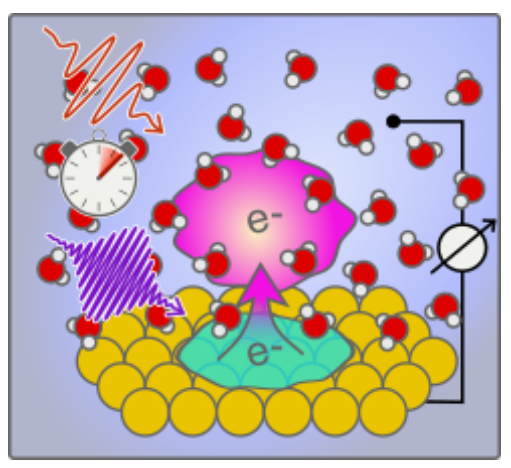

\section{Keywords}

Electrochemistry; Time-resolved spectroscopy; Electron transfer; Hydrated electron; Interfaces 


\section{Supporting Information}

\section{Probing the Birth and Dynamics of Hydrated Electrons at the \\ Gold/Liquid Water Interface via a Novel Optoelectronic}

\section{Approach}

François Lapointe ${ }^{1,2}$, Martin Wolf ${ }^{1}$, R. Kramer Campen ${ }^{1,3}$, Yujin Tong ${ }^{1,3, *}$

\section{Affiliations}

1. Fritz Haber Institute of the Max Planck Society, Faradayweg 4-6, 14195 Berlin, Germany

2. Now at: National Research Council Canada, 1200 Montreal Road, Ottawa, Ontario K1A 0R6, Canada

3. Faculty of Physics, University of Duisburg-Essen, Lotharstrasse 1, 47057 Duisburg, Germany

*Correspondence author: tong@fhi-berlin.mpg.de 


\section{Contents}

S-1 Experimental principles . . . . . . . . . . . . . . . . S-3

S-2 Methodology ............................

S-2.1 Spectroelectrochemical cell $\ldots \ldots \ldots \ldots \ldots \ldots$. . . . . . . . . .

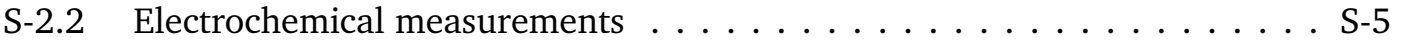

S-2.3 Laser setup . . . . . . . . . . . . . . . . . . . S-6

S-2.3.1 Generation of various wavelengths for the second pump's pulse . S-7

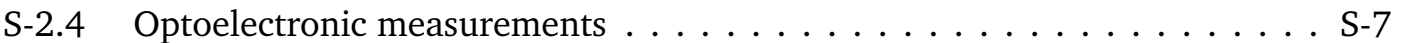

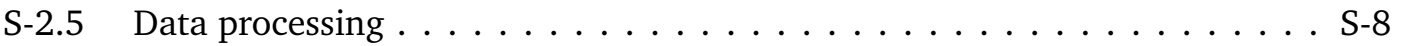

S-2.6 Ultrafast pulse dispersion . . . . . . . . . . . . . . . . S-8

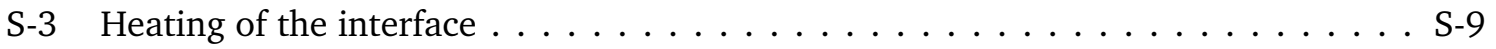

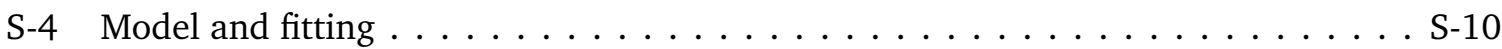

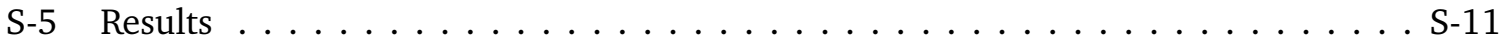

S-5.1 Characterization of the spectroelectrochemical cell . . . . . . . . . S-11

S-5.2 Time delay traces . . . . . . . . . . . . . . . . S -12

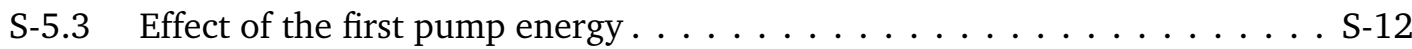

S-5.4 Simulation of heating of the gold electrode surface by an ultrafast UV pulse S-12

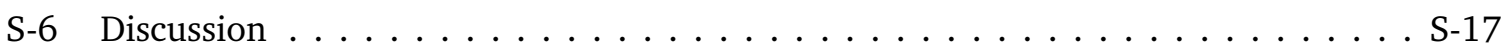

S-6.1 Nature of the photovoltage $\Delta V_{2} \ldots \ldots \ldots \ldots \ldots \ldots \ldots \ldots \ldots \ldots$

S-6.2 Effective spectra . . . . . . . . . . . . . . . . S-19 


\section{S-1 Experimental principles}

This optoelectronic technique to probe the electrode-electrolyte interface combines optical perturbation of the system with coulostatic measurements. As described by Richardson et al., "coulostatic" measurements consist of a perturbation of the electrode-electrolyte interface by an instantaneous pulse of charges [1], followed by monitoring the working electrode (WE) potential in the open circuit potential (OCP) mode of the potentiostat instrument.

Two pulsed beams spatially overlap at the electrode-electrolyte interface while the time averaged photovoltage is continuously monitored with a potentiostat. As the shutter of the first pump beam [ultraviolet (UV), $267 \mathrm{~nm}, \sim 110 \mathrm{fs}$ ] is opened [Fig. S1(a)], carriers are photoexcited in the continuum of electronic states in the metallic electrode, and a portion of the carriers are ejected to the conduction band of water [2]. The combined effect of heat generated at the electrode and photoinjection of carriers to the solution induces a large potential jump $\Delta V_{1}$ on the order of 200-300 mV [inset of Fig. S1(b)] [1, 3, 4].

A potentiostatic variant of the two-pulse technique was used to report on the picosecond dynamics of photoelectrons in hexane [5]. The detection scheme used by Scott was also different: A fast high voltage pulse $(2 \mathrm{kV})$ moved the electrons out from the sample interface to the sensor electrode, $4 \mathrm{~mm}$ away.
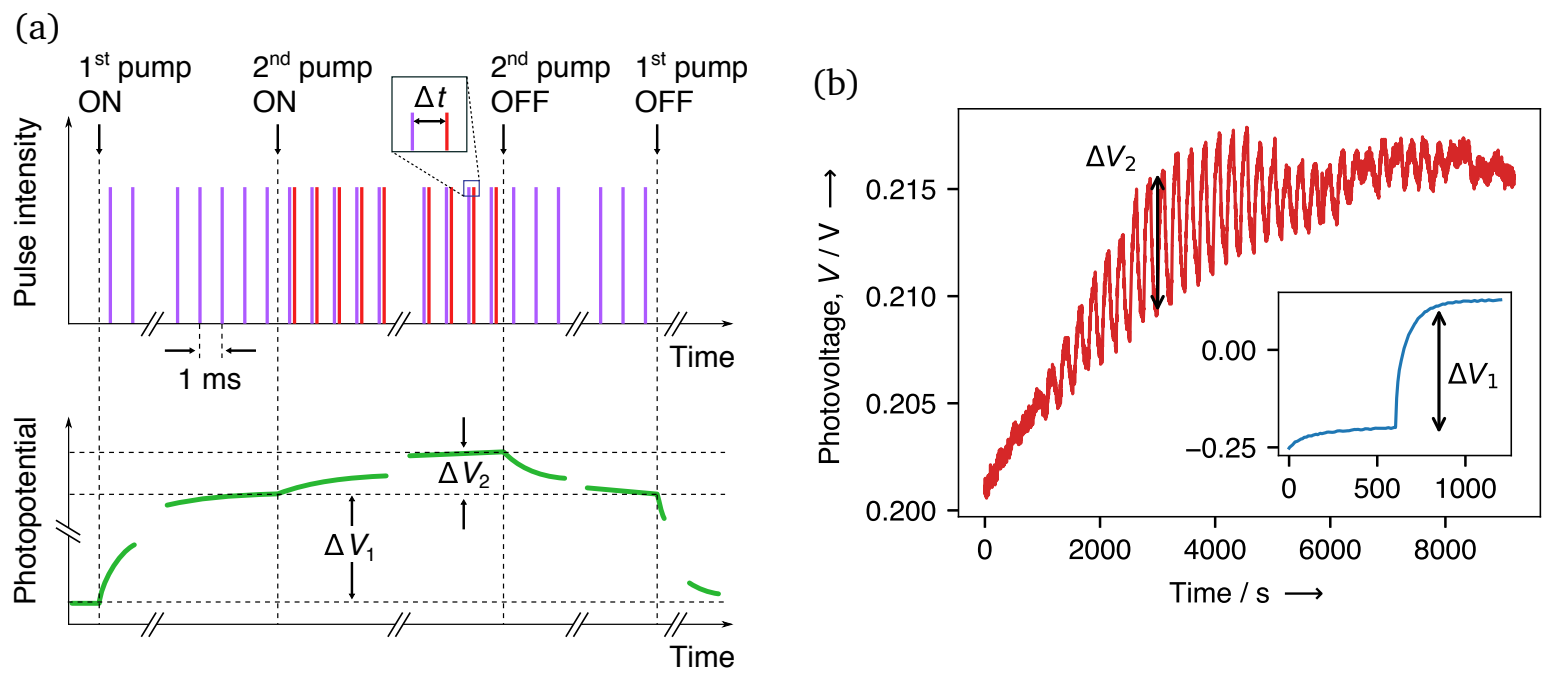

Figure S1: (a) Sequence of pulses for the two pump beams and their effect on the photovoltage represented conceptually. (b) Experimental data of the photovoltage jumps $\Delta V_{2}$ caused by the second pump beam for various delay values. Inset: Photovoltage jump $\Delta V_{1}$ caused by the UV pump beam alone. 
The electrical double layer (EDL) acts as a capacitor with $Q=C V$, where $Q$ is the charge, $C$ is the capacitance of the EDL and $V$ is the voltage. The presence of uncompensated charges in the EDL thus results in a photovoltage. At a concentration of $0.5 \mathrm{M} \mathrm{Na}_{2} \mathrm{SO}_{4}$ in water, the Debye length $\lambda_{\mathrm{D}}$ is $\sim 2.5 \AA$, meaning that we are only probing the charged species located closer than circa two bond lengths from the surface.

As noted above, a voltage can also be established by creating a temperature difference between electrodes. The temperature dependence of the voltage has been exploited previously in temperature jump measurements $[3,6,7]$. The total photovoltage due to heating of the metal solution boundary is the sum of the internal drop of potential at the metal-solution boundary $V_{i}$, of the thermodiffusion potential of the solution (Soret effect) $V_{S}$, and of the thermal EMF of the metal $V_{E M F}$, such that $V=V_{i}+V_{S}+V_{E M F}$. The contribution of $V_{E M F}$ is negligible in comparison to $V_{i}$, but the Soret effect $V_{S}$, while typically much smaller than $V_{i}$, can be important, depending on the electrolyte nature and $\mathrm{pH}$ [3]. Following Benderskii et al.'s method, we estimate that the metal-solution boundary heats up locally by $\sim 180 \mathrm{~K}$ due to the UV pump beam (with a reflection coefficient at $267 \mathrm{~nm}$ of 0.35 , a peak power density of $5.79 \times 10^{9} \mathrm{~W} \mathrm{~cm}^{-2}$, a spot radius of $0.5 \mathrm{~mm}$, a pulse duration of $110 \mathrm{fs}$, and gold's isobaric mass heat capacity of $0.129 \mathrm{~J} \mathrm{~g}^{-1} \mathrm{~K}^{-1}$, thermal conductivity of $0.129 \mathrm{~W} \mathrm{~cm}^{-1} \mathrm{~K}^{-1}$ and specific density of $19.3 \mathrm{~g} \mathrm{~cm}^{-3}$ ) [3]. As shown below using the two-temperature model, heating resulting from an ultrafast pulse can yield a transient electronic temperature $T_{e}$ as high as $1750 \mathrm{~K}$, which results to an increase of the lattice temperature $T_{l}$ on the order of $15 \mathrm{~K}$.

\section{S-2 Methodology}

\section{S-2.1 Spectroelectrochemical cell}

The spectroelectrochemical cell (SEC) consists of a set of 3 gold electrodes ( $5 \mathrm{~nm} \mathrm{Cr} / 200 \mathrm{~nm} \mathrm{Au}$ ) deposited through electron beam deposition on top of a quartz plate ( $\varphi=45 \mathrm{~mm})$ [Fig. S2(a)], thus defining the working, reference and counter electrodes (WE, RE and CE, respectively). The $\mathrm{WE}$ is a disk of $5 \mathrm{~mm}$ diameter at the center of the plate (effective area: $30.8 \mathrm{~mm}^{2}$ ). The CE is larger in area, totalling $51.5 \mathrm{~mm}^{2}$, while the metallic RE (effective area: $10 \mathrm{~mm}^{2}$ ) provides a pseudo-reference. The gap between the $\mathrm{WE}$ and the $\mathrm{CE}$ is $1 \mathrm{~mm}$ and so is the gap between 


\section{(c)}

(a)

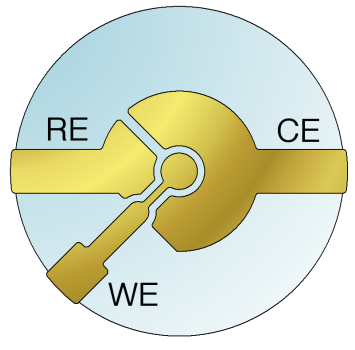

(b)

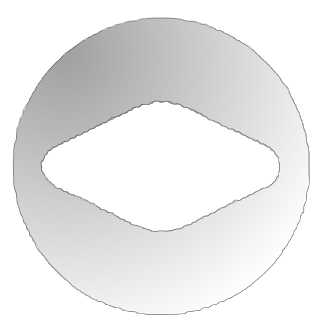

(c)

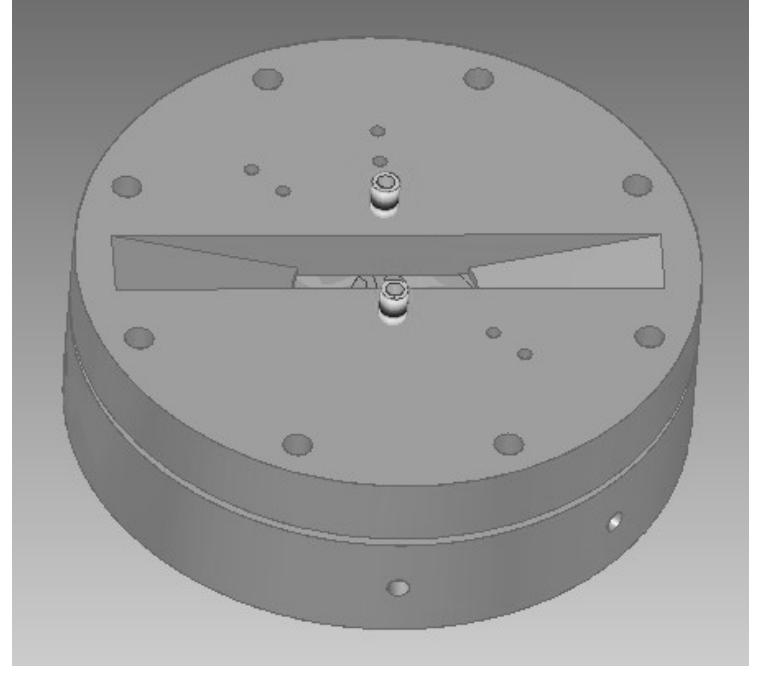

Figure S2: (a) Set of polycrystalline gold electrodes on a quartz plate fabricated by electron beam evaporation. (b) Shape of the $50 \mu \mathrm{m}$ thick PTFE spacer used to defined the inner chamber of the spectroelectrochemical cell yielding a volume of $\sim 6 \mu \mathrm{L}$. (b) The cell assembly showing the aperture for the laser beams, and the inlet / outlet for flowing the electrolyte.

the WE and the RE. The gap between the RE and the CE is $1.5 \mathrm{~mm}$. The cell is closed by capping with a $\mathrm{CaF}_{2}$ window [diameter $25 \mathrm{~mm}$, thickness $3 \mathrm{~mm}$, UV grade, Global Optics (UK) Ltd], drilled through with two holes to allow the flow of the electrolyte, and separated by a $50 \mu \mathrm{m}$ PTFE spacer cut in the shape shown in Fig. S2(b), using the assembly displayed in Fig. S2(c). Copper foil is used to electrically contact the electrodes externally.

The electrolyte consists of $\mathrm{Na}_{2} \mathrm{SO}_{4}$ in deionized water $(18.2 \mathrm{M} \Omega \cdot \mathrm{cm}$, Millipore) at a concentration of $0.5 \mathrm{M}$. It is deaerated by bubbling dry $\mathrm{N}_{2}$ in the reservoir for at least $30 \mathrm{~min}$. before the measurement is started. The flow of the electrolyte is assured by a peristaltic pump at a rate of $6 \mu \mathrm{L} / \mathrm{s}$. In order to avoid any spurious effect by species generated at the CE, the electrolyte inlet is located above the RE and the outlet above the CE.

\section{S-2.2 Electrochemical measurements}

A potentiostat (VSP, Bio-Logic Science Instruments) was employed to record the open circuit potential (OCP). It was found useful, before the coulostatic measurements, to "clean" the WE by performing a series of cyclic voltammetry (CV) sweeps until the CV data showed the appropriate profile for a polycrystalline gold electrode in a thin film configuration. The RE would be cleaned in a similar manner whenever the drift was becoming important. 


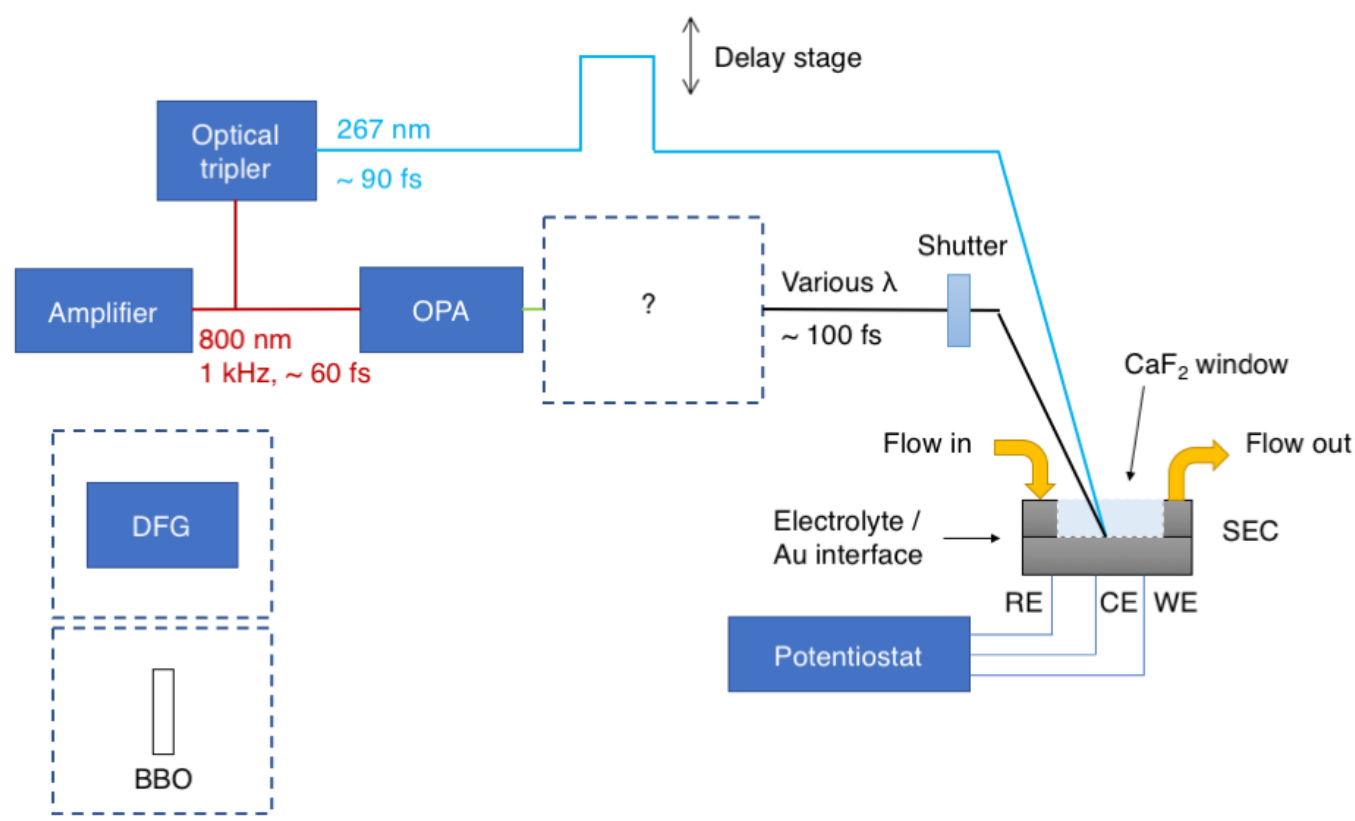

Figure S3: Diagram of the laser setup. The dashed boxed represent the elements that were interchanged as described in $\S \mathrm{S}-2.3 .1$ to generate the various second pump wavelengths. From dispersion calculations presented in § S-2.6 and Fig. S4, the pulse width of the $267 \mathrm{~nm}$ beam is estimated to be $\sim 110 \mathrm{fs}$ at the buried electrode (after transmission through a lens, windows and water layer.

\section{S-2.3 Laser setup}

The layout of the laser system that was employed in this study is shown in [Fig. S3]. In brief, the laser system is composed of a Ti:Sapphire oscillator (Vitara, Coherent) and regenerative amplifier (Legend Elite Duo HE and Cryo PA, Coherent). One third of the amplifier output-7.0 mJ / pulse, $45 \mathrm{fs}$ pulses, $1 \mathrm{kHz}$, centered at $800 \mathrm{~nm}$-was used for the current experiment. $1 \mathrm{~mJ} /$ pulse of this output was used to feed the home-built optical tripler, from which the $267 \mathrm{~nm}$ and $400 \mathrm{~nm}$ photons were generated. A half-wave plate / polarizer / half-wave plate combination was used to adjust the energy of the 267 (400) nm beam at the sample. A typical value of $\sim 4 \mu \mathrm{J} /$ pulse and a lens with a $500 \mathrm{~mm}$ focal length were chosen for the experiments. Depending on the frequency, the second pump beam was either generated from difference frequency generation of Signal and Idler output from a commercial optical parametric amplifier (HE-TOPAS, Light Conversion), or used directly the Idler or Signal or double the frequency of the them.

Synchronization of the arrival of the pulses is necessary. For this purpose, we have used the bleaching by the UV pump of the nonlinear optical signal coming from the gold-electrolyte 
interface. The nonlinear optical signal $\omega_{\mathrm{NL}}=\omega_{1}+\omega_{2}$ stems from a nonresonant sum-frequency generation mixing a $800 \mathrm{~nm} \omega_{1}$ beam with the beam $\omega_{2}$ also serving as a second pump. The bleaching of the optical nonlinear signal is coarsely correlated with the maximum of the photovoltage change due to the second pump pulse. The first pump delay stage is thus swept to find the maximal bleaching.

\section{S-2.3.1 Generation of various wavelengths for the second pump's pulse}

Various ultrashort pulses have been employed in this study as the second pump's pulses. They were generated as following:

670 and $720 \mathrm{~nm}$ The TOPAS' Signal outputs at 1340 and $1440 \mathrm{~nm}$, respectively, were doubled in a $\mathrm{BBO}$ crystal and the fundamental beams were subsequently filtered out.

$800 \mathrm{~nm}$ The $800 \mathrm{~nm}$ residual from the TOPAS after the parametric process was separated from the Idler and Signal beams and attenuated to required energy.

$1000 \mathrm{~nm}$ The TOPAS' Idler output at $2000 \mathrm{~nm}$ was double in a BBO crystal and the fundamental beam was subsequently filtered out.

1333 and $2000 \mathrm{~nm}$ The TOPAS' Signal and Idler beams were directly taken at 1333 and $2000 \mathrm{~nm}$, respectively.

$4000 \mathrm{~nm}$ The TOPAS' Signal and Idler beams were mixed in the DFG crystal $\left(\mathrm{AgGaS}_{2}\right)$ in a noncollinear geometry. The Idler and Signal residuals were spatially filtered out.

\section{S-2.4 Optoelectronic measurements}

After the first pump beam shutter is opened, we let $\Delta V_{1}$ reach an equilibrium value for approximately $10 \mathrm{~min}$ before the time delay series of the second pump beam is started. The shutter of the second pump beam is then sequentially opened and closed at $1 \mathrm{~min}$ intervals and the delay between the pulses of the first and second pumps is stepped at every repetition while photovoltage is continuously acquired in OCP mode. As can be seen in Fig. S1(b), every delay step corresponds to a spike of photovoltage $\Delta V_{2}$ of varying amplitude reaching up to $10 \mathrm{mV}$. Maximum amplitude

occurs in the vicinity of the zero time delay. The spikes are lost in the noise when the $2^{\text {nd }}$ pump 
pulses precede the $1^{\text {st }}$ pump (negative time delays) or when the beams are largely detuned. Whenever the UV pump beam is shut off, the second pump beam does not have any measurable effect on the photovoltage. Such series of delay steps is repeated for every energy of the second pump beam [0.31 (4000), 0.62 (2000), 0.93 (1333), 1.24 (1000), 1.55 (800), 1.72 (720) and $1.85 \mathrm{eV}$ $(670 \mathrm{~nm})]$.

\section{S-2.5 Data processing}

The photovoltage change $\Delta V_{2}$ due to the action of the second pump beam is first extracted in (postmeasurement) data processing from the as-measured photovoltage versus elapsed experimental time [shown in Fig. S1(b)]. It is defined as the difference of the photovoltage measured when the second beam has been impinging for $1 \mathrm{~min}$ to the photovoltage measured after the second pump beam has been shut off for $1 \mathrm{~min}$. Each photovoltage spike thus corresponds to a different delay of the first and second pump pulses. The photovoltage change is then corrected for the measured pulse energy of the first $\left(P_{1}\right)$ and second pump $\left(P_{2}\right)$ beams and the absorptivity of water at the wavelength of the second pump for a given angle $\theta$ of the second pump beam, a water layer

thickness $d_{\mathrm{w}}$ and known water extinction coefficients $\alpha$ [8-10]. The effective water thickness $d_{\mathrm{w}}^{\text {eff }}$ is calculated from Snell's law and the second pump incidence angle in air ( $\theta_{\text {air }}=0.95993$ rad) with $d_{\mathrm{CaF}_{2}}=3 \mathrm{~mm}$ and wavelength-dependent $n_{\mathrm{CaF}_{2}}$ and $n_{\mathrm{w}}[11]$. The different pulse energy values and water extinction coefficients are tabulated in Table S1. The correction to the raw photovoltage change $\Delta V_{2}^{\text {raw }}$ is expressed as:

$$
\Delta V_{2}^{\text {corr }}=\Delta V_{2}^{\text {raw }}\left[P_{1} P_{2} \exp \left(-\alpha d_{\mathrm{w}}^{\text {eff }}\right)\right]^{-1}
$$

It is thus $\Delta V_{2}^{\text {corr }}$ that is presented in the main text as $\Delta V_{2}$ for simplicity.

\section{S-2.6 Ultrafast pulse dispersion}

The effect of $\mathrm{CaF}_{2}$ optics (15 mm lens $+3 \mathrm{~mm}$ window) and water layer (50 $\mu \mathrm{m}$ ) on the UV pulse duration (nominally $60 \mathrm{fs}, 267 \mathrm{~nm}$, at the amplifier's output) was calculated [11-13]. As seen in Fig. S4, the smallest pulse duration is $\sim 106 \mathrm{fs}$. Given that the output from the amplifier is $\sim 60 \mathrm{fs}$, that there should be no significant change of the pulse duration in the tripler, and that the curve 


\begin{tabular}{|l|l||c|c|c|c|}
\hline $\begin{array}{l}\text { Wavelength } \\
\text { of } 2^{\text {nd }} \text { pump } \\
(\mathrm{nm})\end{array}$ & $\begin{array}{l}\text { Photon } \\
\text { energy } \\
(\mathrm{eV})\end{array}$ & $\begin{array}{c}\text { UV pulse } \\
\text { energy } \\
(\mu \mathrm{J})\end{array}$ & $\begin{array}{c}2^{\text {nd }} \text { pulse } \\
\text { energy } \\
(\mu \mathrm{J})\end{array}$ & $\begin{array}{c}\text { Water } \\
\text { abs. coeff. } \\
\left(\mathrm{cm}^{-1}\right)\end{array}$ & Reference \\
\hline \hline 670 & 1.85 & 5.3 & 4 & 0.00439 & {$[8]$} \\
720 & 1.72 & 5.3 & 4 & 0.01231 & {$[8]$} \\
800 & 1.55 & 5 & 8 & 0.02 & {$[9]$} \\
1000 & 1.24 & 4 & 6 & 0.36 & {$[9]$} \\
1333 & 0.93 & 4 & 5.1 & 1.4 & {$[9]$} \\
2000 & 0.62 & 4 & 3.85 & 67 & {$[9]$} \\
4000 & 0.31 & 5 & 4 & 170 & {$[10]$} \\
\hline
\end{tabular}

Table S1: Pulse energy for the first (UV) and second pumps, and the absorption coefficients of water at the second pump wavelength.

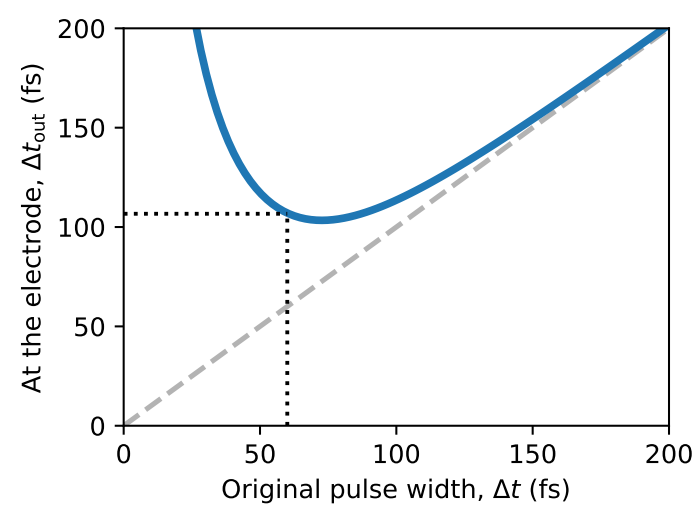

Figure S4: Broadening of the UV pulse after traversal of $15 \mathrm{~mm} \mathrm{CaF}_{2}$ lens, a $3 \mathrm{~mm} \mathrm{CaF}_{2}$ window and $50 \mu \mathrm{m}$ of water.

shown in Fig. S4 is rather flat between 60 and 110 fs, a pulse FWHM of 110 fs for the UV light pulse duration is reasonable and will be used in data analysis and simulations.

\section{S-3 Heating of the interface}

Heating of the gold electrode has been simulated numerically using a two-temperature model (TTM) in Python 3 [14]. The code was tested against the data and numerical simulations from Eesley et al. from nonequilibrium electron heating in copper [15].

We used a gold thickness of $200 \mathrm{~nm}$, a thermal conductivity of $317 \mathrm{~W} \mathrm{~m}^{-1} \mathrm{~K}^{-1}$ [16] and $1 \mathrm{~W} \mathrm{~m}^{-1} \mathrm{~K}^{-1}$ for the electron and lattice systems, respectively, an electron heat capacity of $T_{e} \times$ $3.6 \times 10^{-3} \mathrm{~J} \mathrm{~g}^{-1} \mathrm{~K}^{-1}[16]$ and a lattice heat capacity of $0.129 \mathrm{~J} \mathrm{~g}^{-1} \mathrm{~K}^{-1}$. Gold density was taken as $19.3 \mathrm{~g} \mathrm{~cm}^{-3}$ and the electron-phonon coupling term was $2.2 \times 10^{10} \mathrm{~W} \mathrm{~cm}^{-3} \mathrm{~K}^{-1}$ [17]. 


\section{S-4 Model and fitting}

Aiming to model the dynamics at the interface, we used a system of five coupled ordinary differential equations that are solved numerically to find the populations $N_{i}$ at various states $\mathrm{S}_{0}$ to $\mathrm{S}_{4}$ in the system [Fig. 3(a)]:

$$
\begin{gathered}
\frac{\mathrm{d} N_{0}}{\mathrm{~d} t^{\prime}}=-I\left(t^{\prime}\right) N_{0}+\frac{N_{1}}{\tau_{\mathrm{th}}}, \\
\frac{\mathrm{d} N_{1}}{\mathrm{~d} t^{\prime}}=I\left(t^{\prime}\right) N_{0}-\frac{N_{1}}{\tau_{\mathrm{th}}}-\frac{N_{1}}{\tau_{0}}, \\
\frac{\mathrm{d} N_{2}}{\mathrm{~d} t^{\prime}}=\zeta \frac{N_{1}}{\tau_{0}}-\frac{N_{2}}{\tau_{1}}, \\
\frac{\mathrm{d} N_{3}}{\mathrm{~d} t^{\prime}}=(1-\zeta) \frac{N_{1}}{\tau_{0}}+\frac{N_{2}}{\tau_{1}}-\frac{N_{3}}{\tau_{2}}, \\
\frac{\mathrm{d} N_{4}}{\mathrm{~d} t^{\prime}}=\frac{N_{3}}{\tau_{2}}-\frac{N_{4}}{\tau_{3}},
\end{gathered}
$$

where and $I(t)$ is the intensity of the time-dependent UV pump pulse. In order to correctly capture the dynamics of the shoulder feature in Fig. 1(c), where some amplitude appears early, we follow the concept used by Stähler et al. where a minority portion of the injected electrons reaches directly a trap state [18]. In equations S4 and S5, $\zeta$ is thus the ratio of electrons injected directly to the state $\mathrm{S}_{2}$.

Assuming that only electron populations on the solution side contribute to the signal, the photovoltage change $\Delta V_{2}$ is related to populations $N_{2}, N_{3}$ and $N_{4}$ through absorption coefficients $a_{i}$ :

$$
\Delta V_{2}=a_{2} N_{2}+a_{3} N_{3}+a_{4} N_{4}
$$

Only four parameters are thus adjusted for every excitation energy: three absorption coefficients and a delay offset.

Metals dynamics have been taken into account in the model, even though it was found to be mostly insensitive to them: We have therefore lumped the metal-side thermalization into an effective relaxation time $\tau_{\text {th }}=1 \mathrm{ps}$ [19]. The characteristic times $\tau_{0}=0.12 \mathrm{ps}$ and $\tau_{1}=0.14 \mathrm{ps}$, respectively assigned to injection and initial trapping, have been determined while fitting the fastest decay at an excitation energy of $0.31 \mathrm{eV}$. Considering that the excitation pulse width has 
been taken as $0.11 \mathrm{ps}$ after calculation of the dispersion in the media it traverses, $\tau_{0}$ and $\tau_{1}$ values are likely to be limited by the experimental time resolution. Nonetheless, $\tau_{1}$ can't possibly be much smaller than the excitation pulse width, otherwise the contribution from light absorption by state $S_{2}$ would not be observed. Finally, the characteristic time $\tau_{2}=0.82$ ps related to the final

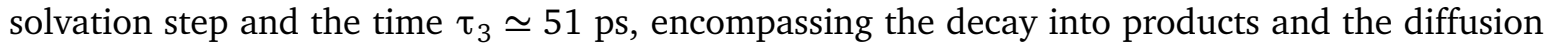
away from the EDL, have been extracted from the fit of the trace corresponding to an excitation energy of $1.85 \mathrm{eV}$. Time resolution is limited by the determination of time zero, which is lying within the convolution of the excitation (110 fs) and the second pulses (65 fs).

Back capture by the electrode [orange arrow in Fig. 3(a)] has not been explicitly implemented but it is expected to contribute to the effective characteristic times. As the back capture rate is higher for electrons of higher energy, the contribution should be more important for $\tau_{0}$ and $\tau_{1}$.

\section{S-5 Results}

\section{S-5.1 Characterization of the spectroelectrochemical cell}

Figure S5 shows a cyclic voltammogram (CV) of the gold electrode in $0.5 \mathrm{M} \mathrm{Na}_{2} \mathrm{SO}_{4}$ aqueous solution collected with a scan rate of $1 \mathrm{mV} / \mathrm{s}$ in the spectroelectrochemical cell.

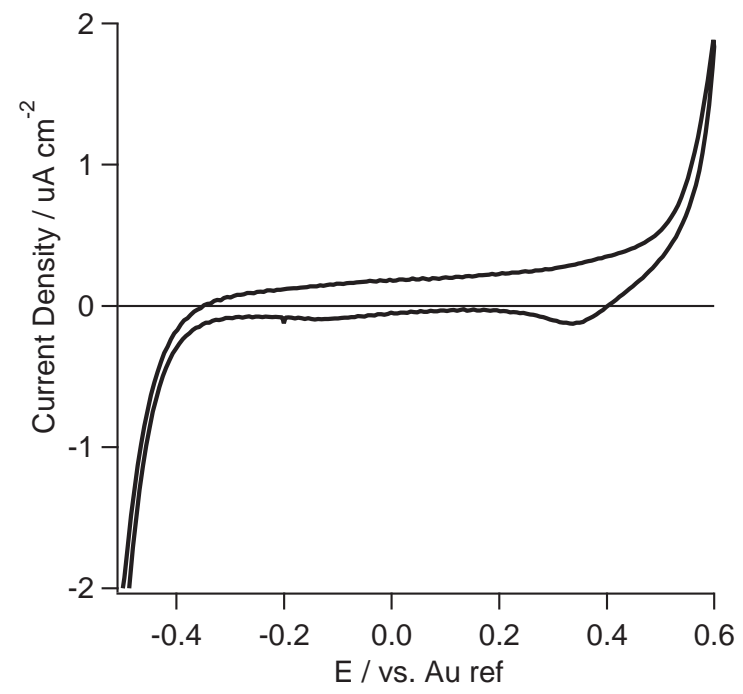

Figure S5: Cyclic voltammogram of the gold electrode in $0.5 \mathrm{M} \mathrm{Na}_{2} \mathrm{SO}_{4}$ in spectroelectrochemical cell (scanning speed $1 \mathrm{mV} / \mathrm{s}$ ). 
Table S2: Characteristic times used in the fitting of the model.

$\begin{array}{ll}\tau_{0} & 0.12 \mathrm{ps} \\ \tau_{1} & 0.14 \mathrm{ps} \\ \tau_{2} & 0.82 \mathrm{ps} \\ \tau_{3} & 51.0 \mathrm{ps} \\ \text { UV pulse FWHM } & 0.11 \mathrm{ps}\end{array}$

\section{S-5.2 Time delay traces}

The full set of time delay traces for various second pump energies is presented in Fig. S6. A zoom near the origin is shown for all energies in Fig. S6(a), while longer traces are displayed in (b) for energies 1.24 to $1.85 \mathrm{eV}$. The fit results (red dashed lines, computed as described in section S-4) are overlaid on the data (gray circles). As described in the main text, the photovoltage response $\Delta V_{2}$ to the UV excitation (first pump) is highly dependent on the second pump wavelength. At low energies, the signal rise and decay is fast, on the order of $100 \mathrm{fs}$. As the second pump energy is increased, the signal persists for much longer, with residual intensity at 100 ps at $1.55 \mathrm{eV}$ and above. We also note the large changes in peak $\Delta V_{2}$. At $0.31 \mathrm{eV}$, the signal reaches approximately $0.18 \mathrm{mV}$, while the peak signal at $1.85 \mathrm{eV}$ is more than 1000 times weaker.

\section{S-5.3 Effect of the first pump energy}

Fig. S7 compares on the same scale the untreated response to a $0.31 \mathrm{eV}$ second pump for two different first pump energies: $3.10 \mathrm{eV}$ (blue, top) and $4.64 \mathrm{eV}$ (UV, bottom). Both traces display an initial drift, but settle to a different photovoltage. The red bands indicate when the second pump shutter is open, with a clear difference between the blue and UV first pumps. Indeed, for the blue first pump, the second pump shutter has a negligible effect on the signal, while a clear photovoltage increase is measured in the case of the UV first pump.

\section{S-5.4 Simulation of heating of the gold electrode surface by an ultrafast UV pulse}

We have simulated the heating of the gold-water interface as described in section S-3 using the two-temperature model and calculated the temperature-related changes due to intraband and interband transitions. Results for both $T_{e}$ and $T_{l}$ are displayed in Fig. S8(a). The UV pulse interaction with the gold surface creates a transient hot electron population with $T_{e}$ rising up to 
(a)

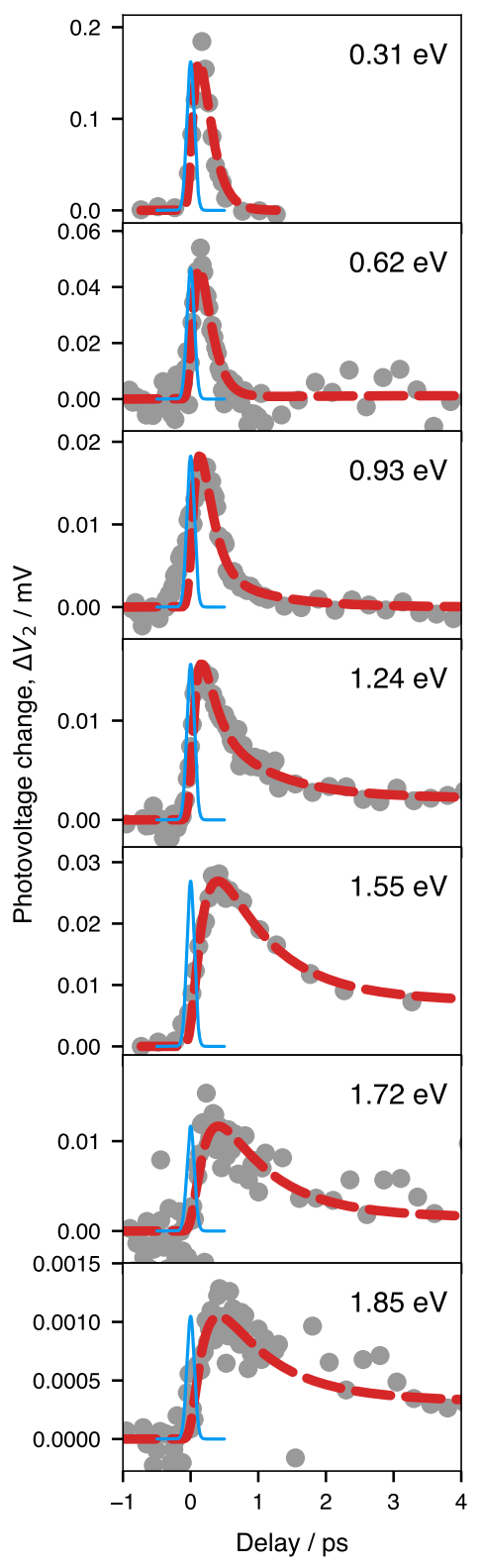

(b)
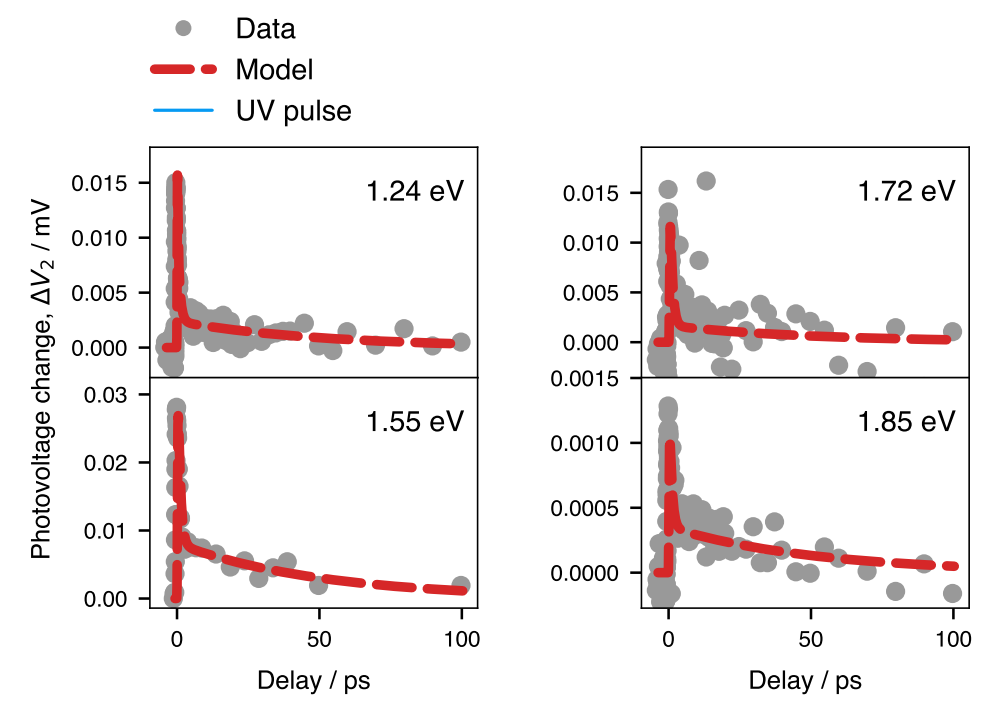

Figure S6: (a-b) The photovoltage change $\Delta V_{2}$ versus the delay time. (a) At delays smaller than 4 ps. (b) At longer delays, up to 100 ps. 


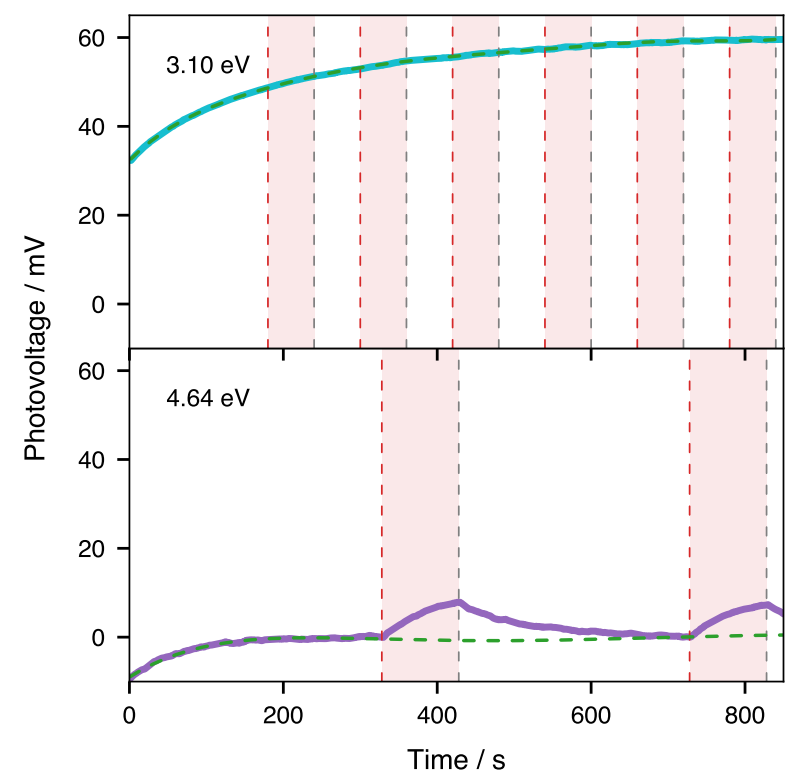

Figure S7: Comparison of the photovoltage when the first excitation pulse is changed from UV (4.64 eV, bottom) to blue ( $3.10 \mathrm{eV}$, top). The second pulse's energy is $0.31 \mathrm{eV}$ in both cases. The red boxes show the times during which the shutter is open and the second pulse impinges on the electrode. The green dashed lines show the polynomial baseline subtracted to give the data shown in Fig. 1 of the main text.

(a)

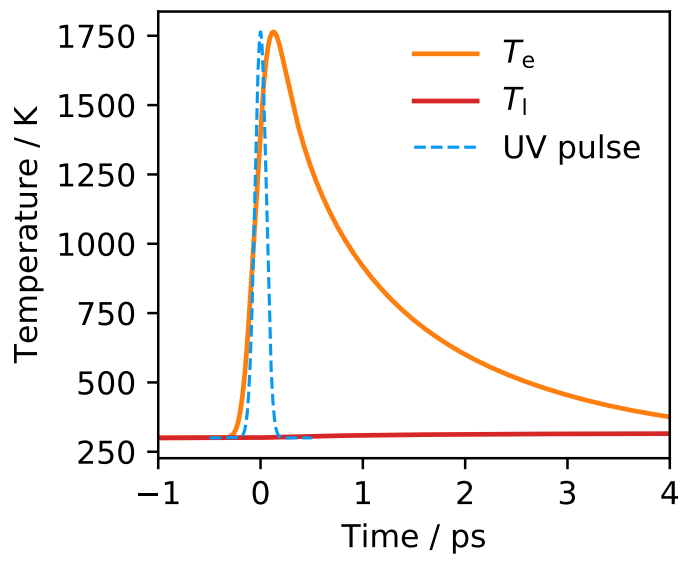

(b)

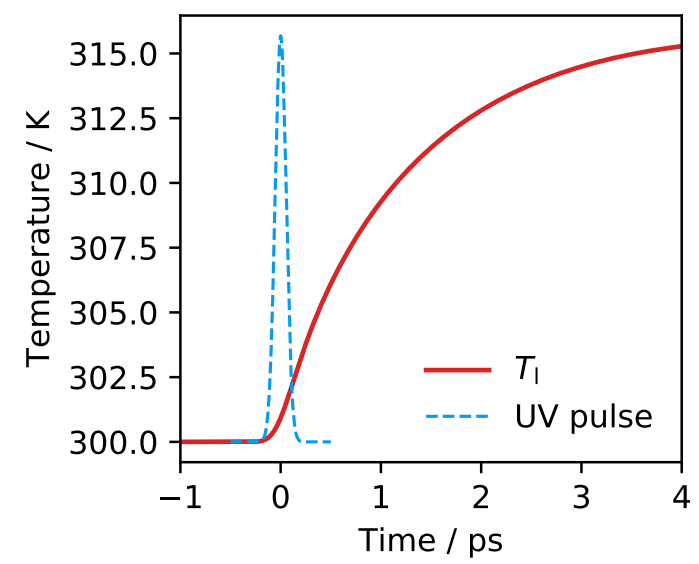

Figure S8: Electronic and lattice temperatures calculated from finite element analysis for an incoming UV pulse $(267 \mathrm{~nm})$ with 110 fs Gaussian FWHM and a peak power density of $5.79 \times$ $10^{9} \mathrm{~W} \mathrm{~cm}^{2}$ on a $200 \mathrm{~nm}$ thick gold film. (a) Electronic and lattice temperatures. (b) Lattice temperature only. 
(a)

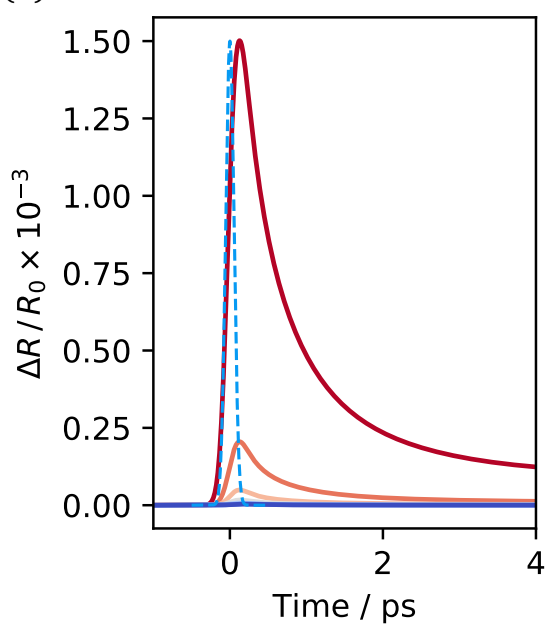

(b)

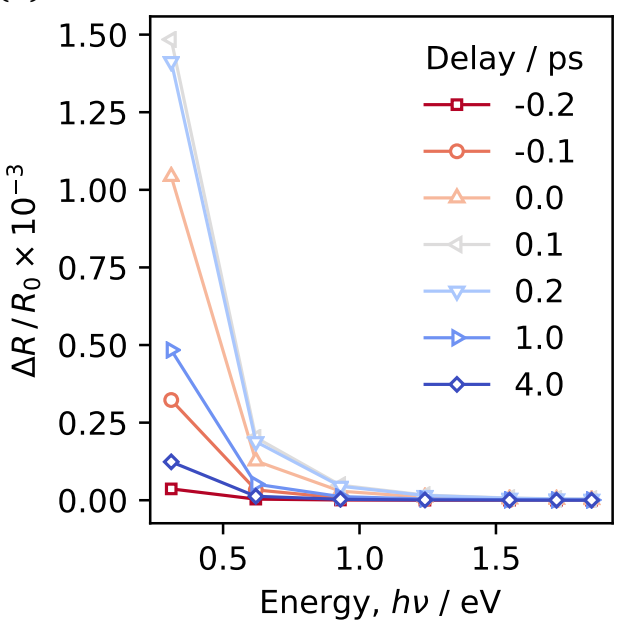

Figure S9: Calculated change in reflectivity due to intraband transitions in gold. (a) As a function of delay time. (b) As a function of photon energy for selected delay times.

$1750 \mathrm{~K}$. The electron system's temperature $T_{e}$ nevertheless rapidly decreases as the hot electrons diffuse into the substrate and scatter with the lattice's phonons. Upon this action, the latter's temperature $T_{l}$ rises by about $15 \mathrm{~K}$ in $4 \mathrm{ps}$. It is noteworthy that neither temperature profile matches directly the delay-dependent $\Delta V_{2}$ traces (Fig. S6.

The reflectivity change $\Delta R / R_{0}$ due to temperature-dependent Drude-like intraband transitions has been modeled according to Block et al. (main text and supplementary materials) [20]. The simulations results are presented in Fig. S9 with the evolution as function of delay in (a) and as a function of second pump energy in (b). We note from (a) that the general time-dependent $\Delta R / R_{0}$ trace is similar to $T_{e}$ in Fig. S8(a). Also, $\Delta R / R_{0}$ is much larger at lower energies, as can be expected from intraband transitions.

Similarly, we have computed the change in Fermi-Dirac (FD) electron distribution $\left(\Delta f / f_{0}\right)$ as a function of temperature (Fig. S10). In order to relate $\Delta f / f_{0}$ to the optical response due to interband transitions, a detailed knowledge of gold's complex permittivity in the $k$-space would be necessary. Nevertheless, the interband transition probability should be dependent on the FD electron distribution and we use here the readily available $\Delta f / f_{0}$ parameter to represent the temporal changes in the system. From Fig. S10(a), we can see that $\Delta f / f_{0}$ rapidly increases upon excitation, but decays almost as fast. In this case, the larger change can be seen at higher energies 
(a)

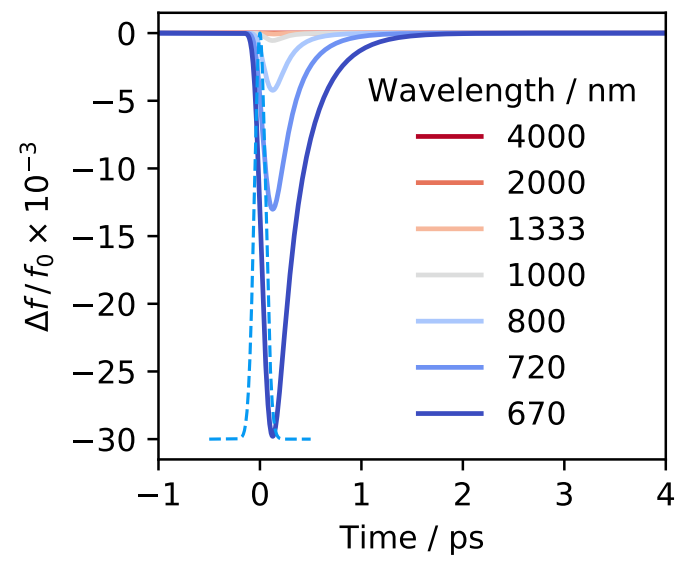

(b)

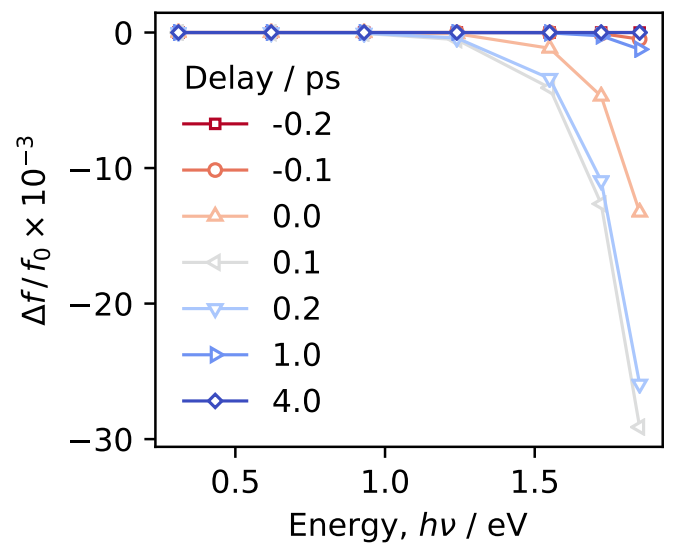

Figure S10: Calculated change (smearing) in Fermi-Dirac electron distribution in gold due to a perturbation by a UV pulse. (a) As a function of delay time for given wavelengths. (b) As a function of photon energy for selected delay times.

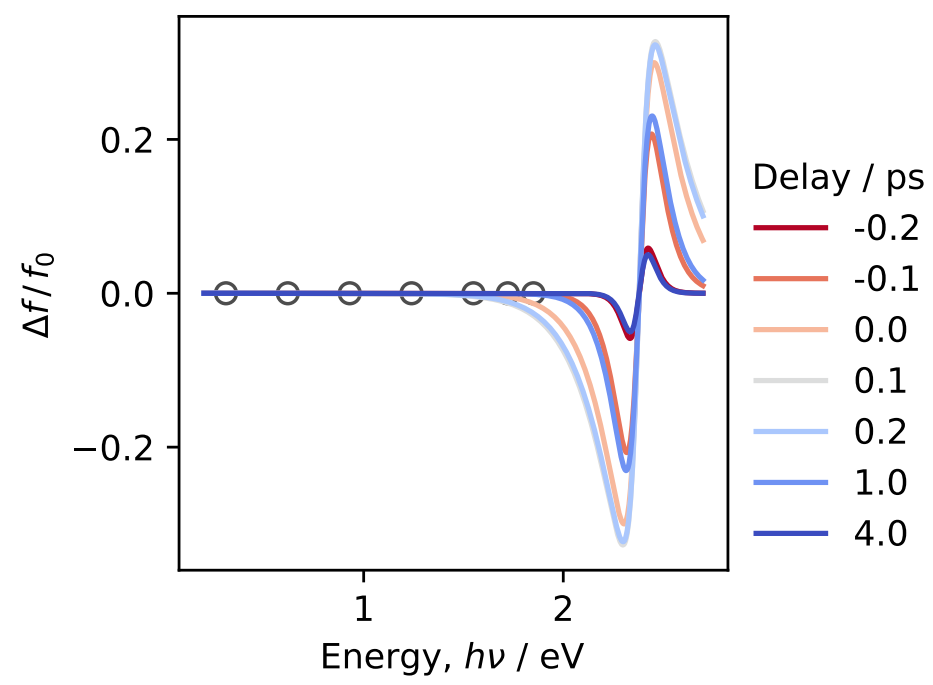

Figure S11: Calculated change (smearing) in Fermi-Dirac electron distribution in gold due to a perturbation by a UV pulse for a broad range of photon energy. The circles show the position, in energy, of this study's data. 
[Fig S10(b)]. Fermi smearing is indeed maximal just above and below the interband threshold $(\sim 2.38 \mathrm{eV})$, yielding the typical "first derivative" shape (Fig. S11).

\section{S-6 Discussion}

\section{S-6.1 Nature of the photovoltage $\Delta V_{2}$}

We discuss here four hypotheses for the origin of the photovoltage $\Delta V_{2}$ and we expose arguments to rule out the first three: $i$. further photoinjection from the electrode, $i i$. photoejection of carriers from the aqueous interface to the electrode, iii. heat originating at the metal surface via metal's electronic system excitation, and $i v$. heat generated at the aqueous side of the interface via resonant absorption by interfacial species.

$i$. In the first hypothesis, we consider the possibility of injecting more electrons in the electrolyte from the population of hot electrons in the electrode through the action of the second laser pulse. However, this effect would follow the relaxation dynamics of hot nonequilibrium electron population in gold, which thermalization time is about 1 ps [19]. The expected dynamics thus do not correspond to the observations where a long-lasting signal persists up to $100 \mathrm{ps.}$

ii. The second hypothesis addresses the reverse process of $i$., namely the photon-triggered return of hot carriers from the electrolyte interface to the electrode, in analogy to charge transfer inverse photoemission spectroscopy (CTRIPS). This possibility can be ruled out because the depletion from the EDL of carriers of the same charge as those photoinjected in the first place would mark a reversal of sign of the photovoltage deltas. Yet, the observations show $\Delta V_{2}$, induced by the second pump, to be of the same sign as $\Delta V_{1}$ which is caused by the first pump.

iii. The third hypothesis assumes that the elevated temperature of the metal electrode surface induces a change in the metal absorptivity that increases the amount of heat generated upon excitation with the second pump. This change in metal absorptivity can come from the perturbation of three types of optical transitions: intraband transitions, normal interband transitions, and low energy interband transitions. Firstly, elevated $T_{e}$ and $T_{l}$ modify the reflectivity related to intraband transitions through an increase of the effective electron scattering rate and decrease of the free carrier density [see Fig. S9(a) and (b)][20]. Temperature-induced changes in intraband transitions affect mainly the lower energy portion of the spectrum, with a monotonous decrease 
towards higher energy; a Drude-like model does not afford any mechanism to account for a shoulder appearing at higher energy. Moreover, the expected dynamics of the intraband contributions is a sharp initial transient due to the nonequilibrium hot electron population, and a persisting signal due to the long-lasting effect of the heat transferred to the lattice $\left(T_{l}\right)$. To the contrary, our observations show a rapid rise and fall of the signal at 0.31 and $0.62 \mathrm{eV}$, with no residual after $\sim 500$ fs [Fig. 1(a), main text]. Secondly, perturbation of the normal interband transitions of gold results in a differential spectral shape reflecting the change of electron occupancy[21]. The electron occupancy change is itself described by the Fermi smearing induced by the first excitation pump [see Fig S10(a) and (b)]. Fermi smearing, however, can not explain the sloping of the spectra from the infrared to the visible, nor the presence of a shoulder at $1.55 \mathrm{eV}$ [Fig. 1(c), main text]. Nevertheless, the reversal of the sloping of the spectra at short delays [reddish traces, Fig. 1(c), main text] in comparison to the spectra at longer delays cannot be explained by Fermi smearing, in which case a smooth decay of the signal concomitant with a shift towards higher energy would be expected. We instead observe a signal that seems to peak in the THz region and traverses the measurement range to settle in the visible region. Thirdly, depletion of the metal valence states opens the possibility of low energy interband transitions from the $d$ band to the $s$ band. The spectral response of these low energy interband transitions should evolve inversely to the intraband transitions, where the lower energy transitions will be quenched before the higher energy ones due to the energy dependence of the electron-electron scattering time [22]. We have not been able to rule out this possible contribution to the signal, nor the concurrent evolution of intraband and normal interband transitions. A proper modeling of the gold dielectric function response to an excitation pulse with respect to time would be required to understand their contribution. While it is not possible to rule out this possible contribution to the signal, no previous studies have shown metal dynamics similar to Fig. 1(c) (main text). Furthermore, as discussed above, replacing the $267 \mathrm{~nm}$ first pump with a $400 \mathrm{~nm}$ pump completely quenches the ultrafast response at the interface [Fig. 2(b), main text].

This leads to $i v$. the resonant excitation of species present at the interface, resulting in an increase in the amount of heat generated at the metal/solution boundary. In bulk water, the excess electron frequency-dependent dynamics are characterized by a high energy absorption band centered around $1.72 \mathrm{eV}(720 \mathrm{~nm})$ and by a low energy absorption band peaking in the 
terahertz region. Moreover, as the localized electrons lose kinetic energy in the first few hundreds of femtoseconds, the size of its box shrinks down, and its $p \leftarrow s$ transition is displaced towards higher energies to attain an equilibrium point, which is in bulk water around $1.72 \mathrm{eV}(720 \mathrm{~nm})$.

\section{S-6.2 Effective spectra}

The spectrum $a_{2}$ [top panel of Fig. 4(c), main text] corresponding to the hot electron population shows a profile that rises strongly at low energies. It can be best fitted by a Lorentzian peak shape centered at 0 with a full width at half maximum (FWHM) of $(0.2 \pm 0.1) \mathrm{eV}$. The spectrum of the trapped electron population $a_{3}$ [middle panel of Fig. 4(c), main text] exhibits a peak shape that can be described almost equally as well by a Lorentzian or Gaussian function, centered at $(1.51 \pm 0.03) \mathrm{eV}$ with a FWHM of $(0.31 \pm 0.05) \mathrm{eV}$ and $(0.38 \pm 0.02) \mathrm{eV}$, respectively. Similarly, fitting the spectrum of the solvated electron population $a_{4}$ [bottom panel of Fig. 4(c), main text] yields a center position of $(1.45 \pm 0.03) \mathrm{eV}$ and a FWHM of $(0.1 \pm 0.1) \mathrm{eV}$ for the Lorentzian function, and $(1.47 \pm 0.02) \mathrm{eV}$ and $(0.27 \pm 0.03) \mathrm{eV}$ for the Gaussian function, the latter matching better the amplitude of the spectrum $a_{4}$.

\section{References}

[1] J. H. Richardson, S. B. Deutscher, A. S. Maddux, J. E. Harrar, D. C. Johnson, W. L. Schmelzinger, S. P. Perone, Journal of Electroanalytical Chemistry and Interfacial Electrochemistry 1980, 109, 95-114.

[2] Y. Y. Gurevich, Y. V. Pleskov, Z. A. Rotenberg, Photoelectrochemistry, Springer US, Boston, MA, 1980.

[3] V. A. Benderskii, G. I. Velichko, Journal of Electroanalytical Chemistry 1982, 140, 1-22.

[4] J. F. Smalley, C. V. Krishnan, M. Goldman, S. W. Feldberg, I. Ruzic, Journal of Electroanalytical Chemistry 1988, 248, 255-282.

[5] T. W. Scott, Journal of Physical Chemistry 1986, 90, 1739-1741.

[6] J. F. Smalley, L. Geng, S. W. Feldberg, L. C. Rogers, J. Leddy, Journal of Electroanalytical Chemistry 1993, 356, 181-200.

[7] V. Climent, B. A. Coles, R. G. Compton, Journal of Physical Chemistry B 2001, 105, 1066910673.

[8] R. M. Pope, E. S. Fry, Applied Optics 1997, 36, 8710.

[9] J. A. Curcio, C. C. Petty, Journal of the Optical Society of America 1951, 41, 302. 
[10] H. D. Downing, D. Williams, Journal of Geophysical Research 1975, 80, 1656-1661.

[11] M. N. Polyanskiy, Refractive index database, Accessed on 2019-08-15. https://refractiveindex.info.

[12] J. C. Diels, W. Rudolph, Ultrashort Laser Pulse Phenomena 2nd ed., P. F. Liao, P. Kelley (Eds.), Academic Press, Inc., Burlington, 2006.

[13] The Effect of Dispersion on Ultrashort Pulses, Accessed on 2019-08-15. https://www.newport.com/n/the-effect-of-dispersion-on-ultrashort-pulses.

[14] L. Alber, S. Valentino, U. Vivek, B. Stefano, udcm-su/heat-diffusion-1D: NTMpy-package, 2019, DOI: 10.5281/ZENODO.3333493. https : //zenodo.org/record/3333493.

[15] G. L. Eesley, Physical Review B 1986, 33, 2144-2151.

[16] A. N. Smith, P. M. Norris, Applied Physics Letters 2001, 78, 1240-1242.

[17] A. Jain, A. J. McGaughey, Physical Review B 2016, 93, 081206(R).

[18] J. Stähler, J.-C. Deinert, D. Wegkamp, S. Hagen, M. Wolf, Journal of the American Chemical Society 2015, 137, 3520-3524.

[19] W. S. Fann, R. Storz, H. W. K. Tom, J. Bokor, Physical Review B 1992, 46, 13592-13595.

[20] A. Block, M. Liebel, R. Yu, M. Spector, Y. Sivan, F. J. García de Abajo, N. F. van Hulst, Science Advances 2019, 5, eaav8965.

[21] R. W. Schoenlein, W. Z. Lin, J. G. Fujimoto, G. L. Eesley, Physical Review Letters 1987, 58, $1680-1683$.

[22] C.-K. Sun, F. Vallée, L. Acioli, E. P. Ippen, J. G. Fujimoto, Physical Review B 1993, 48, 365-368. 
